\title{
Generic two-loop Higgs mass calculation from a diagrammatic approach
}

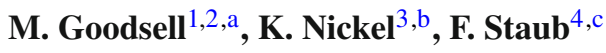 \\ ${ }^{1}$ LPTHE, UMR 7589, UPMC Univ Paris 06, Sorbonne Universités, 75005 Paris, France \\ ${ }^{2}$ CNRS, LPTHE, UMR 7589, 75005 Paris, France \\ ${ }^{3}$ Bethe Center for Theoretical Physics and Physikalisches Institut der Universität Bonn, Nußallee 12, 53115 Bonn, Germany \\ ${ }^{4}$ Theory Division, CERN, 1211 Geneva 23, Switzerland
}

Received: 30 March 2015 / Accepted: 1 June 2015 / Published online: 26 June 2015

(C) The Author(s) 2015. This article is published with open access at Springerlink.com

\begin{abstract}
We calculate the corrections to the Higgs mass in general theories restricted to the case of massless gauge bosons (the gaugeless limit). We present analytic expressions for the two-loop tadpole diagrams, and corresponding expressions for the zero-momentum limit of the Higgs self-energies, equivalent to the second derivative of the twoloop effective potential. We describe the implementation in SARAH, which allows for an efficient, accurate and rapid evaluation for generic theories. In the appendix, we provide the expressions for the tadpole diagrams in the case of massive gauge bosons.
\end{abstract}

\section{Introduction}

With the discovery of the Higgs boson in the range of 125$126 \mathrm{GeV}$ the standard model (SM) has been completed [1,2]. The uncertainty in the Higgs mass measurement has continuously decreased and is well below $0.5 \mathrm{GeV}$ today [3]. This small uncertainty is currently much better than the theoretical prediction in any scenario beyond the standard model (BSM). Therefore, more precise calculations are necessary to better confront BSM models with the Higgs mass measurement. This has two motivations of particular weight: (1) In the minimal supersymmetric standard model (MSSM) and many extensions thereof, radiative corrections are required to be at least as significant as the tree-level contribution, so higher-order corrections are especially important. (2) In the Standard Model and non-supersymmetric extensions thereof a precise calculation is required to extract the parameters of the model, which when run to high energies gives information

\footnotetext{
a e-mail: goodsell@lpthe.jussieu.fr

be-mail: nickel@th.physik.uni-bonn.de

c e-mail: florian.staub@cern.ch
}

as regards the stability or lifetime of the potential—which may point the way to new physics if, as currently seems to be the case, the potential is metastable. Beyond these motivations, for a generic model of new physics with boundary conditions fixed from the top down (such as supersymmetric models) it is important to know what regions of parameter space are allowed, compatible with the measured Higgs mass. For example, a one-loop calculation may naively lead to excluding certain constrained scenarios, whereas with a two-loop calculation the Higgs mass may be large enough; this is related to the difficulty in estimating the error in the Higgs mass calculation, since at two-loop order there are new contributions from particles that have no direct couplings to the Higgs, and a simple variation of the renormalisation scale as an estimate of the error is not sufficient.

In general, there are three approaches to tackle the problem of finding the Higgs mass precisely: (i) effective potential calculations, (ii) diagrammatic calculations, (iii) renormalisation group equation methods. We shall concentrate in the following on the first two options. Calculations from the effective potential suffer from a larger uncertainty compared to diagrammatic calculations because of the missing momentum contributions. However, these are only really pronounced at one-loop level, and it is already possible to calculate the full one-loop Higgs mass including momentum dependence for generic models using SARAH [4-8] to produce SPheno $[9,10]$ output or SOFTSUSY [11] output via FlexibleSUSY [12]; explicit results have been known for some time for specific models such as the MSSM with real parameters [13-16] and complex parameters [17-19], and for the NMSSM with real parameters [20-22] and complex parameters [23]. At two loops the momentum effects are expected to be small: according to recent calculations for specific models they are comparable to the experimental 
uncertainty [24-26] and since the momentum-dependent corrections due to new physics scale at best as $m_{H}^{2} / M_{\text {New Physics }}^{2}$ relative to the effective potential contribution, we expect this to be a general result. Hence effective potential calculations, with their concomitant great simplification over the diagrammatic approach, should be useful at two-loop order and beyond (even if the inclusion of the momentum dependence will ultimately be necessary to reach the experimental accuracy).

In general even a two-loop calculation of the dominant contributions at zero external momentum is available for just two supersymmetric models: the MSSM [2743] and partially for the next-to-minimal supersymmetric standard model (NMSSM) with real [20] and complex [44] parameters. There continues to be much work in this direction and there are now some calculations of the strong (i.e. proportional to $\alpha_{\mathrm{s}}$ ) momentum-dependent contributions for the MSSM [24-26]. These results have variously been made available to the community in modelspecific public codes: FeynHiggs [45], Soft SUSY [11], SuSpect [46] and SPheno [9,10] for the MSSM and NMSPEC [47], Next-to-Minimal SOFTSUSY [48,49], and NMSSMCALC [50] for the NMSSM. There are also some three-loop results, in the Standard Model [51,52] and the MSSM [53,54] with the code H3m based on [54].

The state of the art in these calculations is, however, somewhat suprising given that the complete generic expressions for the two-loop effective potential, valid for a general renormalisable quantum field theory, have been available for more than ten years by the work of Martin [55]. These were applied to a complete two-loop calculation of the light Higgs mass in the MSSM in the effective potential approach in Ref. [39]. Furthermore, generic results for the diagrammatic calculation including the momentum dependence up to leading order in gauge couplings have been available in the literature for almost as long [56-58]. Unfortunately the results of Ref. [39] suffered from the so-called "Goldstone boson catastrophe" (recently re-explored in $[59,60]$ ) due to the presence of tachyons in the tree-level spectrum so were numerically unstable. Perhaps due to this no public code was made available to exploit these prior to Ref. [61] where an implementation in SARAH/SPheno was presented. Currently, the only generic two-loop results relevant for the Higgs mass calculation still not present in the literature are the all-electroweak loops and the corrections to the $Z$ boson mass relevant for determining the electroweak expectation value. These will be the subject of future work. Here we shall instead continue the process started in Ref. [61] of making the pioneering generic results of Martin available in a public code-which entails performing some new calculations.

As we stated above, calculating the two-loop corrections to the Higgs mass in the effective potential approach is expected to be a good approximation. However, there is more than one way to actually perform even this calculation: either we can calculate the potential and numerically take the derivatives, as done in Refs. $[38,61]$, or we can perform the calculation diagrammatically and set the external momentum to zero. In this work we shall exploit this equivalence: we shall analytically take the derivatives of the effective potential, producing expressions equivalent to the diagrammatic calculation and having the same structure, but with much simpler loop functions. The advantages of this over the first method are that the results are numerically stable ${ }^{1}$ it is in principle a faster computation for more complicated models where the numerical method must make several passes to ensure stability; and it can later be extended to a full diagrammatic calculation by simply changing the loop functions-but at zero momentum the loop functions are much simpler and therefore significantly faster to evaluate. We shall therefore compute the analytic expressions for the first and second derivatives of the two-loop effective potential and implement them in SARAH. As in Refs. [61,62] we shall ignore broken gauge groups and adopt the same ansätze regarding the contribution of the electroweak gauge couplings to the tree-level Higgs mass matrix; the reasons for restricting to the so-called "gaugeless limit" are: (a) partial circumvention of the Goldstone boson catastrophe (complete evasion in the case of the MSSM or any theory where the electroweak gauge couplings entirely determine the Higgs quartic potential); (b) significant simplification in the expressions and therefore speed in calculation; (c) the electroweak contributions are expected to be small, of the same magnitude as the momentum-dependent contributions. On the other hand, in the appendix we provide just the tadpole contributions in the case of broken gauge groups, and we will return to the full expressions in future work.

The layout of the paper is as follows. In Sect. 2 we explain our procedure to take the derivatives of the effective potential to extract the two-loop tadpole functions for a general theory with massless gauge bosons in a form convenient for automation. In Sect. 2.3 we summarise our results for the tadpole diagrams; we present the full set of second derivatives in Appendix B. The implementation of these results in SARAH, including some technical details of the translation of the generic results into an algorithm, is explained in Sect. 3 before we conclude in Sect. 4. Impatient readers interested in using our implementation of the results might want to jump directly to Sect. 3.2.

\footnotetext{
1 That is, not subject to potential errors from ill-judged step sizes in the numerical derivation or from parameters being too small.
} 


\section{Derivatives of the effective potential with massless vectors}

In this section we derive the expressions for the two-loop tadpoles in a general quantum field theory with massless gauge bosons in Landau gauge. To do this, we analytically take the derivatives of the expressions in [55]. Writing the couplings in the notation of that paper, the theory is defined by real scalars $\phi_{i}$, Weyl fermions $\psi_{I}$ and massless gauge bosons $A_{\mu}^{a}$ where the gauge covariant derivative for the fermions and scalars are

$$
\begin{aligned}
& D_{\mu} \psi_{I} \equiv \partial_{\mu} \psi_{I}+i g A_{\mu}^{a}\left(T^{a}\right)_{I}^{J} \psi_{J}, \\
& D_{\mu} \phi_{i} \equiv \partial_{\mu} \phi_{i}+i g A_{\mu}^{a} \theta_{i j}^{a} \phi_{j} .
\end{aligned}
$$

The structure constants $\theta_{i j}^{a}$ are imaginary antisymmetric matrices that obey the gauge algebra but, since we are writing in terms of real bosonic fields, for complex representations they will have twice the dimension of the equivalent generators $T^{a}$ (so e.g. a $U(1)$ generator is two-dimensional). We define as usual $\operatorname{tr}\left(\theta^{a} \theta^{a}\right)=\sum_{i} d(i) C(i)$ where $d(i)$ is the dimension of the representation of field $i$ and $C(i)$ the quadratic Casimir, and similarly for $T^{a}$.

The Lagrangian is then composed of the normal kinetic terms of the scalars and fermions using the above covariant derivatives supplemented by purely scalar and scalar-fermion interactions

$\mathcal{L}_{S}=-\frac{1}{6} \lambda^{i j k} \phi_{i} \phi_{j} \phi_{k}-\frac{1}{24} \lambda^{i j k l} \phi_{i} \phi_{j} \phi_{k} \phi_{l}$

$\mathcal{L}_{S F}=-\frac{1}{2} y^{I J k} \psi_{I} \psi_{J} \phi_{k}+$ c.c.

$y$ is in general a dimensionless complex tensor with $y^{I J k}=$ $y^{J I k}$, while $\lambda^{i j k}, \lambda^{i j k l}$ are real, symmetric tensors.<smiles>C1CCCCCCCCCCCCCCC1</smiles>

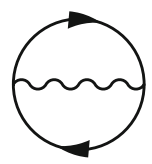

$S S$ $F F V$

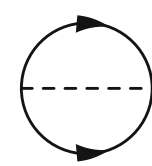

FFS

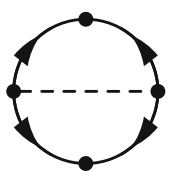

$\overline{F F} S$

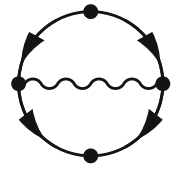

$\overline{F F} V$

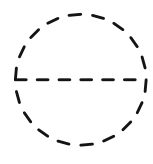

$S S S$

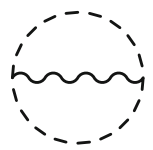

$S S V$
Fig. 1 Two-loop diagrams contributing to the effective potential in the gaugeless limit

\subsection{Effective potential}

We can simplify the expression for the effective potential in the Landau gauge given in Ref. [55] for our case; with all gauge groups unbroken some diagrams do not contribute. The non-vanishing diagrams are shown in Fig. 1.

The contribution of each diagram to the effective potential is given by

$$
\begin{aligned}
V_{S S S}^{(2)} & =\frac{1}{12}\left(\lambda^{i j k}\right)^{2} f_{S S S}\left(m_{i}^{2}, m_{j}^{2}, m_{k}^{2}\right) \\
V_{S S}^{(2)} & =\frac{1}{8} \lambda^{i i j j} f_{S S}\left(m_{i}^{2}, m_{j}^{2}\right) \\
V_{F F S}^{(2)} & =\frac{1}{2}\left|y^{I J k}\right|^{2} f_{F F S}\left(m_{I}^{2}, m_{J}^{2}, m_{k}^{2}\right) \\
V_{\overline{F F} S}^{(2)} & =\frac{1}{4} y^{I J k} y^{I^{\prime} J^{\prime} k} M_{I I^{\prime}}^{*} M_{J J^{\prime}}^{*} f_{\overline{F F} S}\left(m_{I}^{2}, m_{J}^{2}, m_{k}^{2}\right) \\
V_{S S V}^{(2)} & =\frac{g^{2}}{4} d(i) C(i) f_{S S V}\left(m_{i}^{2}, m_{i}^{2}, 0\right) \\
V_{F F V}^{(2)} & =\frac{g^{2}}{2} d(I) C(I) f_{F F V}\left(m_{I}^{2}, m_{I}^{2}, 0\right) \\
V_{\overline{F F} V}^{(2)} & =-\frac{g^{2}}{2} d(I) C(I) m_{I}^{2} f_{\overline{F F} V}\left(m_{I}^{2}, m_{I}^{2}, 0\right)
\end{aligned}
$$

Here, $y$ and $\lambda$ are the trilinear and quartic couplings of Eq. (2.2), $g$ is a gauge coupling, and $M$ are fermion masses. The loop functions, given in terms of standard basis functions given in Appendix $\mathrm{A}$, are the same for $\overline{M S}$ and $\overline{D R}^{\prime}$ in the case of

$$
\begin{aligned}
f_{S S S}= & -I(x, y, z), \\
f_{S S}= & J(x, y), \\
f_{F F S}= & J(x, y)-J(x, z)-J(y, z)+(x+y-z) I(x, y, z), \\
f_{\overline{F F} S}= & 2 I(x, y, z), \\
f_{S S V}= & (x+y)^{2}+3(x+y) I(x, y, 0)+3 J(x, y), \\
& -2 x J(x)-2 y J(y)
\end{aligned}
$$

but differ for those with vectors and fermions:

\begin{tabular}{|c|c|c|}
\hline & $\overline{M S}$ & $\overline{D R}^{\prime}$ \\
\hline$f_{F F V}$ & 0 & $-(x+y)^{2}+2 x J(x)+2 y J(y)$ \\
$f_{\overline{F F} V}$ & $6 I(x, y, 0)+2(x+y)-4 J(x)-4 J(y)$ & $6 I(x, y, 0)$ \\
\hline
\end{tabular}

All of these functions are symmetric on the substitution of their first two indices, but may not be so with the third. In fact, we can then combine the vector-fermion diagrams to give

$V_{F F V}^{(2)}+V_{\overline{F F V}}^{(2)} \equiv \frac{g^{2}}{2} d(I) C(I) F_{F V}\left(m_{I}^{2}\right)$ 
where

$F_{F V}(x) \equiv-4 x^{2}+4 x J(x)-6 x I(x, x, 0)+\delta_{M S} 4 x J(x)$

where $\delta_{M S}$ is zero for $\overline{D R}^{\prime}$ and one for $\overline{M S}$.

\subsection{Derivatives of the potential}

Here we shall analytically take the derivatives of the potential. In a generic model, we may want the derivatives of the Higgs potential in terms of some unrotated fields, i.e. in a basis where the mass matrix is not diagonal; let us say that we start with such a case. We write the tree-level potential in terms of some expectation values $\hat{v}_{i}$ and the associated real fluctuations $S_{i}^{0}$ as

$$
\begin{aligned}
V^{\text {scalar tree }}= & V_{0}\left(\hat{v}_{i}\right)+\frac{1}{2} \hat{m}_{i j}^{2} S_{i}^{0} S_{j}^{0}+\frac{1}{6} \hat{\lambda}_{0}^{i j k} S_{i}^{0} S_{j}^{0} S_{k}^{0} \\
& +\frac{1}{24} \hat{\lambda}_{0}^{i j k l} S_{i}^{0} S_{j}^{0} S_{k}^{0} S_{l}^{0} .
\end{aligned}
$$

Then there is a tree-level rotation

$S_{i}^{0}=R_{i j}^{0} S_{j}$

to diagonalise the mass matrix; we then obtain

$$
\begin{aligned}
V^{\text {scalar tree }}= & V_{0}+\frac{1}{2} m_{i}^{2} S_{i} S_{i}+\frac{1}{6} \lambda^{i j k} S_{i} S_{j} S_{k} \\
& +\frac{1}{24} \lambda^{i j k l} S_{i} S_{j} S_{k} S_{l} .
\end{aligned}
$$

We can write $\phi_{i}=v_{i}+S_{i}$ and work with the couplings of Eq. (2.2). We then need the quantities which enter in the effective potential calculation, which are masses and couplings depending on $\left\{S_{i}\right\}$.

In general, we have

$$
\begin{aligned}
m_{i j}^{2}(S) & =\frac{\partial^{2}}{\partial S_{i} \partial S_{j}} V \\
& =m_{i}^{2} \delta_{i j}+\lambda^{i j k} S_{k}+\frac{1}{2} \lambda^{i j k l} S_{k} S_{l}, \\
\lambda^{i j k}(S) & =\frac{\partial^{3}}{\partial S_{i} \partial S_{j} \partial S_{k}} V \\
& =\lambda^{i j k}+\lambda^{i j k l} S_{l} \\
\lambda^{i j k l}(S) & =\lambda^{i j k l}
\end{aligned}
$$

with the shorthand notation $S \equiv\left\{S_{i}\right\}$. Hence we can write

$$
\begin{aligned}
\frac{\partial}{\partial S_{r}} \lambda^{i j k l}(S) & =0, \\
\frac{\partial}{\partial S_{r}} \lambda^{i j k}(S) & =\lambda^{i j k r}(S), \\
\frac{\partial}{\partial S_{r}} m_{i j}^{2}(S) & =\lambda^{i j r}+\lambda^{i j k r} S_{k} .
\end{aligned}
$$

Similarly for the fermions we have

$$
\begin{aligned}
& m_{I}^{2} \delta_{J}^{I}=M^{I I^{\prime}} M_{J I^{\prime}}^{*} \\
& \rightarrow \frac{\partial}{\partial S_{r}} M^{I I^{\prime}} M_{J I^{\prime}}^{*}=y^{I I^{\prime} r} M_{J I^{\prime}}^{*}+M^{I I^{\prime}} y_{J I^{\prime} r} \\
& \frac{\partial}{\partial S_{r}} y^{I J s}=0 .
\end{aligned}
$$

However, for the purposes of the effective potential, we then require a further diagonalisation for $m_{i j}^{2}(S)$ : we put

$S_{i}=R_{i j}(S) S_{j}^{\prime}$

We name the couplings in this basis as $\tilde{m}_{i}(S), \tilde{\lambda}_{S}^{i j k}(S), \tilde{\lambda}^{i j k l}$ $(S)$. We then express the effective potential by inserting the couplings and masses in the basis $\left\{S_{i}^{\prime}\right\}$ into the formulae of Eqs. (2.3)-(2.9). However, to take the derivatives we rewrite the expressions in terms of the basis $\left\{S_{i}\right\}$, and use the trick (with $\mathbf{m}^{2} \equiv\left(m_{i j}^{2}\right)$ )

$$
\frac{\partial}{\partial S_{r}}\left(\frac{1}{q^{2}+\mathbf{m}^{2}}\right)_{i j}=-\left(\frac{1}{q^{2}+\mathbf{m}^{2}}\right)_{i k} \frac{\partial m_{k k^{\prime}}^{2}}{\partial S_{r}}\left(\frac{1}{q^{2}+\mathbf{m}^{2}}\right)_{k^{\prime} j} .
$$

For similar expressions we write by abuse of notation (using $\left.C \equiv 16 \pi^{2} \mu^{2 \epsilon}(2 \pi)^{-d}[57]\right)$

$\mathbf{J}\left(m_{i k}^{2}, m_{j l}^{2}\right) \equiv C^{2} \int d^{d} q d^{d} k\left(\frac{1}{q^{2}+\mathbf{m}^{2}}\right)_{i k}\left(\frac{1}{k^{2}+\mathbf{m}^{2}}\right)_{j l}$.

For fermion propagators we can write

$$
\frac{M_{I I^{\prime}}}{q^{2}+m_{I}^{2}} \rightarrow M_{I J} \frac{1}{q^{2}+M^{J K} M_{K I^{\prime}}}=\frac{1}{q^{2}+M_{I J} M^{J K}} M_{K I^{\prime}}
$$

Let us demonstrate our method on a brief example:

$$
\begin{aligned}
& \frac{\partial}{\partial S_{p}^{0}} \frac{1}{8} \tilde{\lambda}^{i i j j} f_{S S}\left(\tilde{m}_{i}^{2}, \tilde{m}_{j}^{2}\right)=R_{r p}^{0} \frac{\partial}{\partial S_{r}} \frac{1}{8} \tilde{\lambda}^{i i j j} f_{S S}\left(\tilde{m}_{i}^{2}, \tilde{m}_{j}^{2}\right) \\
&= R_{r p}^{0} \frac{\partial}{\partial S_{r}} \frac{1}{8} \lambda^{i k j l} f_{S S}\left(m_{i k}^{2}, m_{j l}^{2}\right) \\
&= R_{r p}^{0} \frac{1}{4} \lambda^{i k j l}(S) f_{S S}^{(1,0)} \\
& \times\left(m_{i m}^{2}, m_{n k}^{2} ; m_{j l}^{2}\right) \frac{\partial}{\partial S_{r}}\left(m_{m n}^{2}\right) \\
&= R_{r p}^{0} \frac{1}{4} \lambda^{i k j l}(S) f_{S S}^{(1,0)} \\
& \quad \times\left(m_{i m}^{2}, m_{n k}^{2} ; m_{j l}^{2}\right) \lambda^{m n r}(S) \\
& \underset{S \rightarrow 0}{\longrightarrow} R_{r p}^{0} \frac{1}{4} \lambda^{i k j j} \lambda^{i k r} f_{S S}^{(1,0)}\left(m_{i}^{2}, m_{k}^{2} ; m_{j}^{2}\right) .
\end{aligned}
$$


Recall that $f_{S S}(x, y) \equiv J(x, y)$ where $J$ is the finite loop function and $\mathbf{J}(x)=J(x)-\frac{x}{\epsilon}$; see Eq. (A.3). Here we have defined

$$
\begin{aligned}
f_{S S}^{(1,0)}(x, y ; z) \equiv & -C^{2} \int d^{d} k d^{d} q \frac{1}{k^{2}+x} \frac{1}{k^{2}+y} \frac{1}{q^{2}+z} \\
& +\frac{C}{\epsilon}\left(\mathbf{J}(z)-C z \int d^{d} k \frac{1}{k^{2}+x} \frac{1}{k^{2}+y}\right)+\frac{z}{\epsilon^{2}} \\
= & -C^{2}\left(\int d^{d} k \frac{1}{k^{2}+x} \frac{1}{k^{2}+y}\right) \\
& \times\left(\int d^{d} q \frac{1}{q^{2}+z}+\frac{z}{\epsilon}\right)+\frac{C}{\epsilon} \mathbf{J}(z)+\frac{z}{\epsilon^{2}} \\
= & \frac{1}{x-y}(\mathbf{J}(x)-\mathbf{J}(y)) J(z)+\frac{1}{\epsilon} J(z) \\
= & \frac{1}{x-y}(J(x)-J(y)) J(z) \\
= & -B_{0}(x, y) J(z) .
\end{aligned}
$$

Note that the $R_{r a}^{0}$ are not functions of $S_{i}$ and so do not present complications if we want to take further derivatives.

We can similarly take the derivatives of all the remaining loop functions; these are derived from the basis

$$
\begin{aligned}
& \frac{\partial}{\partial S_{r}} J\left(m_{i}^{2}, m_{j}^{2}\right) \rightarrow-B_{0}\left(m_{i}^{2}, m_{k}^{2}\right) J\left(m_{j}^{2}\right) \frac{\partial m_{i k}^{2}}{\partial S_{r}} \\
& \quad-J\left(m_{i}^{2}\right) B_{0}\left(m_{j}^{2}, m_{k}^{2}\right) \frac{\partial m_{j k}^{2}}{\partial S_{r}}, \\
& \frac{\partial}{\partial S_{r}} B_{0}\left(m_{i}^{2}, m_{j}^{2}\right) \rightarrow-C_{0}\left(m_{i}^{2}, m_{k}^{2}, m_{j}^{2}\right)\left(\frac{\partial m_{i k}^{2}}{\partial S_{r}}+\frac{\partial m_{j k}^{2}}{\partial S_{r}}\right), \\
& \frac{\partial}{\partial S_{r}} I\left(m_{i}^{2}, m_{j}^{2}, m_{k}^{2}\right) \rightarrow-U_{0}\left(m_{i}^{2}, m_{l}^{2}, m_{j}^{2}, m_{k}^{2}\right) \frac{\partial m_{i l}^{2}}{\partial S_{r}} \\
& \quad-U_{0}\left(m_{j}^{2}, m_{l}^{2}, m_{i}^{2}, m_{k}^{2}\right) \frac{\partial m_{j l}^{2}}{\partial S_{r}} \\
& \quad-U_{0}\left(m_{k}^{2}, m_{l}^{2}, m_{i}^{2}, m_{j}^{2}\right) \frac{\partial m_{k l}^{2}}{\partial S_{r}}
\end{aligned}
$$

and

$$
\begin{aligned}
\frac{\partial}{\partial S_{r}} m_{i}^{2} I\left(m_{i}^{2}, m_{j}^{2}, m_{k}^{2}\right) \rightarrow & \frac{\partial m_{i l}^{2}}{\partial S_{r}} I\left(m_{l}^{2}, m_{j}^{2}, m_{k}^{2}\right) \\
& -m_{i}^{2} \frac{\partial m_{i l}^{2}}{\partial S_{r}} U_{0}\left(m_{l}^{2}, m_{i}^{2}, m_{j}^{2}, m_{k}^{2}\right) \\
& -\frac{\partial m_{j l}^{2}}{\partial S_{r}} m_{i}^{2} U_{0}\left(m_{l}^{2}, m_{j}^{2}, m_{k}^{2}, m_{i}^{2}\right) \\
& -\frac{\partial m_{k l}^{2}}{\partial S_{r}} m_{i}^{2} U_{0}\left(m_{l}^{2}, m_{k}^{2}, m_{i}^{2}, m_{j}^{2}\right) .
\end{aligned}
$$

The derivative of a typical term in the effective potential will have the form

$$
\begin{aligned}
& \frac{\partial}{\partial S_{r}} A^{i j k} A^{i j k} f_{\alpha}\left(x_{i}, y_{j}, z_{k}\right)=2 f_{\alpha}\left(x_{i}, y_{j}, z_{k}\right) A^{i j k} \frac{\partial}{\partial S_{r}}\left(A^{i j k}\right) \\
& \quad+\left\{A^{i j k} A^{i^{\prime} j k} \frac{\partial m_{i i^{\prime}}^{2}}{\partial S_{r}} f_{\alpha}^{(1,0,0)} \times\left(x_{i}, x_{i^{\prime}} ; y_{j}, z_{k}\right)+(x \leftrightarrow y)\right. \\
& \quad+(x \leftrightarrow z)\},
\end{aligned}
$$

where, generalising the above, it is straightforward to show that for a generic function appearing in the effective potential $f_{\alpha}$ composed of polynomials (even containing monomials with negative exponents) multiplying the loop functions above, we can write

$f_{\alpha}^{(1,0,0)}(x, u ; y, z) \equiv \frac{f_{\alpha}(x, y, z)-f_{\alpha}(u, y, z)}{x-u}$

and similarly for permutation of the indices. On the other hand, this explicit expression is often inconvenient in practice due to the need to carefully take the smooth limit when $x=u$; it is instead more practical to rewrite the right-hand side in terms of our basis of loop functions multiplied by suitable polynomials. In the following we present explicit expressions for the first derivatives (and, in the appendix, the second derivatives), which have been appropriately simplified to remove the apparent singularities.

\subsection{First derivatives}

Here we gather the set of two-loop tadpole diagrams. There are only three topologies, two of which only apply to the all-scalar case. All generic possible diagrams are given in Fig. 2. Here we present our results for the first derivatives in the basis $\left\{S_{i}\right\}$, so without the rotation matrices $R$; the full tadpole in the original basis is then given by

$$
\frac{\partial V^{(2)}}{\partial S_{p}^{0}}=R_{r p}^{0}\left[T_{S}+T_{S S F F}+T_{F F F S}+T_{S V}+T_{F V}\right]
$$

with the tadpoles on the right-hand side to be defined below.

\subsubsection{All scalars}

We start with the purely scalar diagrams which are in the first row of Fig. 2. The entire contribution is given by

$T_{S}=T_{S S}+T_{S S S}+T_{S S S S}$

with

$T_{S S}=\frac{1}{4} \lambda^{i k j j} \lambda^{i k r} f_{S S}^{(1,0)}\left(m_{i}^{2}, m_{k}^{2} ; m_{j}^{2}\right)$,
$T_{S S S}=\frac{1}{6} \lambda^{r i j k} \lambda^{i j k} f_{S S S}\left(m_{i}^{2}, m_{j}^{2}, m_{k}^{2}\right)$, 


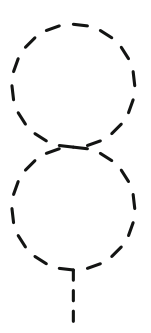

$T_{S S}$

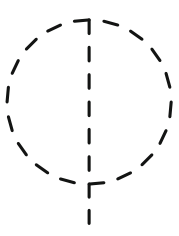

$T_{S S S}$

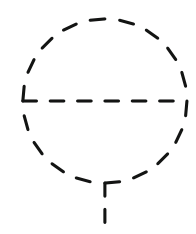

$T_{S S S S}$

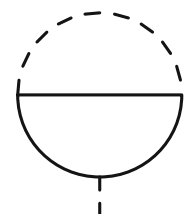

$T_{F F F S}$

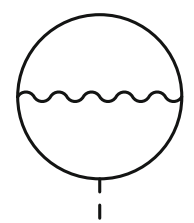

$T_{F V}$

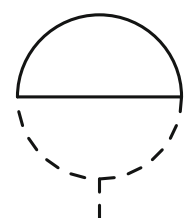

$T_{S S F F}$

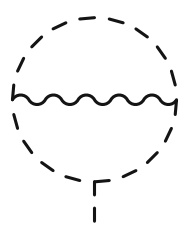

$T_{S V}$
Fig. 2 Tadpole diagrams at the two-loop level which do not vanish in the gaugeless limit

$T_{S S S S}=\frac{1}{4} \lambda^{r i i^{\prime}} \lambda^{i j k} \lambda^{i^{\prime} j k} f_{S S S}^{(1,0,0)}\left(m_{i}^{2}, m_{i^{\prime}}^{2} ; m_{j}^{2}, m_{k}^{2}\right)$.

The new loop functions are defined as

$$
\begin{aligned}
f_{S S}^{(1,0)}(x, y ; z) & \equiv-B_{0}(x, y) J(z), \\
f_{S S S}^{(1,0,0)}(x, y ; u, v) & \equiv U_{0}(x, y, u, v) .
\end{aligned}
$$

Note that $f_{S S}^{(1,0)}$ corresponds to $X_{S S S}, f_{S S S}$ corresponds to $S_{S S S}$, and $f_{S S S}^{(1,0,0)}$ to $W_{S S S S}$ of Ref. [56] in the limit of zero external momentum.

\subsubsection{Scalars and fermions}

We have, first, the diagrams with two scalar propagators:

$$
\begin{aligned}
T_{S S F F}= & \frac{1}{2} y^{I J k} y_{I J l} f_{F F S}^{0,0,1}\left(m_{I}^{2}, m_{J}^{2} ; m_{k}^{2}, m_{l}^{2}\right) \lambda^{k l r} \\
& -\left[\frac{1}{2} y^{I J k} y^{I^{\prime} J^{\prime} k} M_{I I^{\prime}}^{*} M_{J J^{\prime}}^{*} \lambda^{k l r}\right. \\
& \left.\times U_{0}\left(m_{l}^{2}, m_{k}^{2}, m_{I}^{2}, m_{J}^{2}\right)+\text { c.c. }\right] .
\end{aligned}
$$

Then we turn to one scalar and three fermion propagators:

$$
\begin{aligned}
T_{F F F S}= & 2 \operatorname{Re}\left[y^{I J r} y_{I K m} y^{K L m} M_{J L}^{*}\right] \\
& \times T_{F \bar{F} F S}\left(m_{I}^{2}, m_{J}^{2}, m_{K}^{2}, m_{m}^{2}\right) \\
& +2 \operatorname{Re}\left[y_{I J r} y^{I K m} y^{J L m} M_{K L}^{*}\right] \\
& \times T_{F F \bar{F} S}\left(m_{I}^{2}, m_{J}^{2}, m_{K}^{2}, m_{m}^{2}\right) \\
& -2 \operatorname{Re}\left[y^{I J r} y^{K L m} y^{M N m} M_{I K}^{*} M_{J M}^{*} M_{L N}^{*}\right] \\
& \times T_{\overline{F F F} S}\left(m_{I}^{2}, m_{J}^{2}, m_{L}^{2}, m_{m}^{2}\right) .
\end{aligned}
$$

Here we have defined

$$
\begin{aligned}
& \quad f_{F F S}^{1,0,0}\left(m_{I^{\prime}}^{2}, m_{I}^{2}, m_{J}^{2} ; m_{k}^{2}\right) \equiv-B_{0}\left(m_{I^{\prime}}^{2}, m_{I}^{2}\right) J\left(m_{J}^{2}\right) \\
& \quad+B_{0}\left(m_{I^{\prime}}^{2}, m_{I}^{2}\right) J\left(m_{k}^{2}\right)+I\left(m_{I^{\prime}}^{2}, m_{J}^{2}, m_{k}^{2}\right)-\left(m_{I}^{2}+m_{J}^{2}-m_{k}^{2}\right) \\
& \quad \times U_{0}\left(m_{I^{\prime}}^{2}, m_{I}^{2}, m_{J}^{2}, m_{k}^{2}\right), \\
& f_{F F S}^{0,0,1}\left(m_{I}^{2}, m_{J}^{2} ; m_{k}^{2}, m_{l}^{2}\right) \equiv B_{0}\left(m_{l}^{2}, m_{k}^{2}\right) J\left(m_{I}^{2}\right) \\
& \quad+B_{0}\left(m_{l}^{2}, m_{k}^{2}\right) J\left(m_{J}^{2}\right)-I\left(m_{I}^{2}, m_{J}^{2}, m_{l}^{2}\right)-\left(m_{I}^{2}+m_{J}^{2}-m_{k}^{2}\right) \\
& \quad \times U_{0}\left(m_{l}^{2}, m_{k}^{2}, m_{I}^{2}, m_{J}^{2}\right), \\
& T_{\overline{F F F} S}\left(m_{I}^{2}, m_{J}^{2}, m_{L}^{2}, m_{m}^{2}\right) \equiv U_{0}\left(m_{I}^{2}, m_{J}^{2}, m_{L}^{2}, m_{m}^{2}\right), \\
& T_{F \bar{F} F S}\left(m_{I}^{2}, m_{J}^{2}, m_{K}^{2}, m_{m}^{2}\right) \equiv f_{F F S}^{1,0,0}\left(m_{I}^{2}, m_{J}^{2}, m_{K}^{2} ; m_{m}^{2}\right), \\
& T_{F F \bar{F} S}\left(m_{I}^{2}, m_{J}^{2}, m_{K}^{2}, m_{m}^{2}\right) \equiv I\left(m_{I}^{2}, m_{K}^{2}, m_{m}^{2}\right) \\
& \quad-m_{I}^{2} U_{0}\left(m_{I}^{2}, m_{J}^{2}, m_{K}^{2}, m_{m}^{2}\right) .
\end{aligned}
$$

\subsubsection{Diagrams with vectors}

Finally, the two generic diagrams involving vectors are given by

$$
\begin{aligned}
T_{S V}= & \frac{g^{2}}{2} d(i) C(i) \lambda^{i i r}\left(3 I\left(0, m_{i}^{2}, m_{i}^{2}\right)-J\left(m_{i}^{2}\right)+2 m_{i}^{2}\right) \\
= & \frac{g^{2}}{2} d(i) C(i) \lambda^{i i r} m_{i}^{2} \\
& \left.\times\left[-12+11 \log m_{i}^{2} / Q^{2}-3 \log ^{2} m_{i}^{2} / Q^{2}\right)\right], \\
T_{F V}= & g^{2} d(I) C(I) \operatorname{Re}\left(M_{I I^{\prime}} y^{I I^{\prime} r}\right) 4\left(-3 I\left(0, m_{I}^{2}, m_{I}^{2}\right)\right. \\
& \left.+5 J\left(m_{I}^{2}\right)-4 m_{I}^{2}+\delta_{\overline{M S}}\left[2 J\left(m_{I}^{2}\right)+m_{I}^{2}\right]\right) \\
= & g^{2} d(I) C(I) \operatorname{Re}\left(M_{I I^{\prime}} y^{I I^{\prime} r}\right) 4 m_{I}^{2}\left[6-7 \log m_{I}^{2} / Q^{2}\right. \\
& \left.+3 \log ^{2} m_{I}^{2} / Q^{2}+\delta_{\overline{M S}}\left[2 \log m_{I}^{2} / Q^{2}-1\right]\right] .
\end{aligned}
$$

\subsection{Second derivatives}

To find the second derivatives of the potential we can apply the same technique. However, in principle, we can simply use the results of [56], which computed (diagrammatically) the two-loop scalar self-energies at leading order in gauge couplings. Since we want the self-energies for neutral scalars, this comprises all of the contributions, and if we want the 


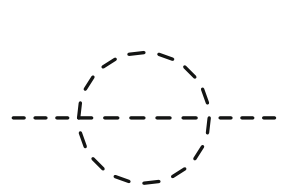

$S_{S S S}$

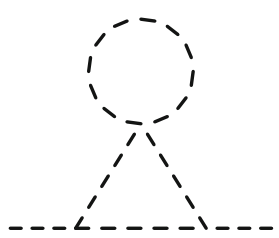

$Y_{S S S S}$

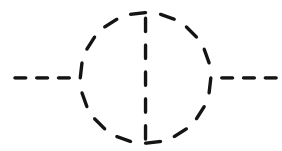

$M_{S S S S S}$

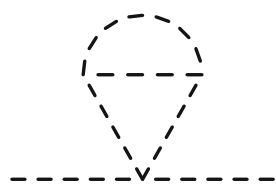

$W_{S S S S}$<smiles>CC1CCC2CCC(C)CCC2CC1</smiles>

$Z_{S S S S}$

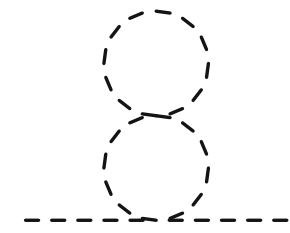

$X_{S S S}$

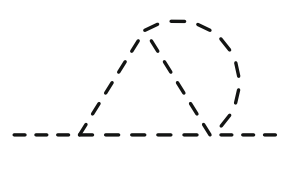

$U_{S S S S}$

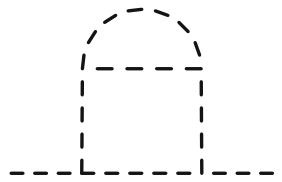

$V_{S S S S S}$

Fig. 3 Two-loop self-energy diagrams involving only scalars

results in the effective potential approach we can simply set the external momenta to zero (and, for this work, the masses of the gauge bosons to zero). In fact, for the majority of the diagrams, these yield the same result. However, in a few cases we find that by taking the derivatives of the potential we find simpler results (which are of course entirely equivalent). The full result is given by

$$
\begin{aligned}
\frac{\partial V^{(2)}}{\partial S_{p}^{0} \partial S_{q}^{0}}= & R_{i p}^{0} R_{j q}^{0} \Pi_{i j}(0) \\
\equiv & R_{i p}^{0} R_{j q}^{0}\left[\Pi_{i j}^{S}+\Pi_{i j}^{S F(W)}+\Pi_{i j}^{S F_{4}(M)}+\Pi_{i j}^{S_{2} F_{3}(M)}\right. \\
& \left.+\Pi_{i j}^{S_{3} F_{2}(V)}+\Pi_{i j}^{S F_{4}(V)}+\Pi_{i j}^{S V}+\Pi_{i j}^{F V}\right]
\end{aligned}
$$

where the superscripts correspond to the numbers of scalars, fermions and vectors with types of topology listed for diagrams $(M)$ and $(V)$. We give the complete set of relevant expressions in Appendix B; the corresponding diagrams are shown in Figs. 3 and 4. The expressions for $\Pi_{i j}^{S F_{4}(V)}, \Pi_{i j}^{S V}$ and $\Pi_{i j}^{F V}$ exhibit particular simplifications in our approach.

\section{Implementation in SARAH}

We have implemented the new routines in SARAH. By including the first and second derivatives of the effective potential using the analytic expressions here, rather than numerically taking the derivatives of the couplings and masses as performed in the previous version [61], we can guarantee greater numerical stability, accuracy and speed improvements. Moreover, this approach allows for a straightforward upgrade to the pole mass calculation by simply changing the loop functions called to those defined in Ref. [56] based on loop functions implemented in TSIL [63], which will be made possible in a future version.
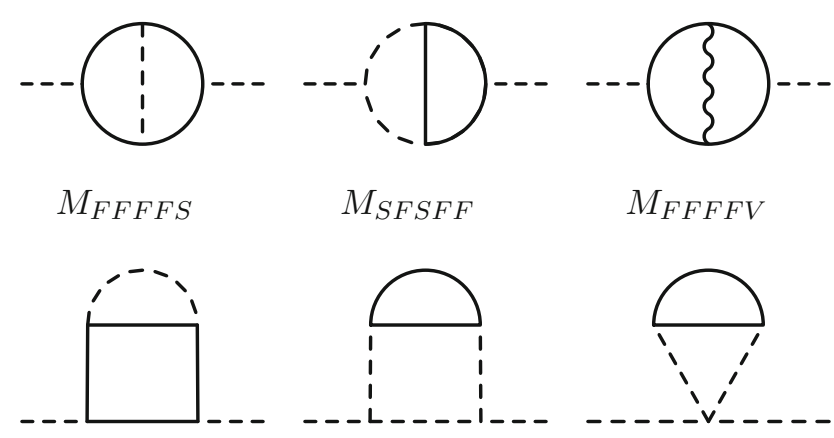

$M_{S F S F F}$

$M_{F F F F V}$

$V_{F F F F S}$
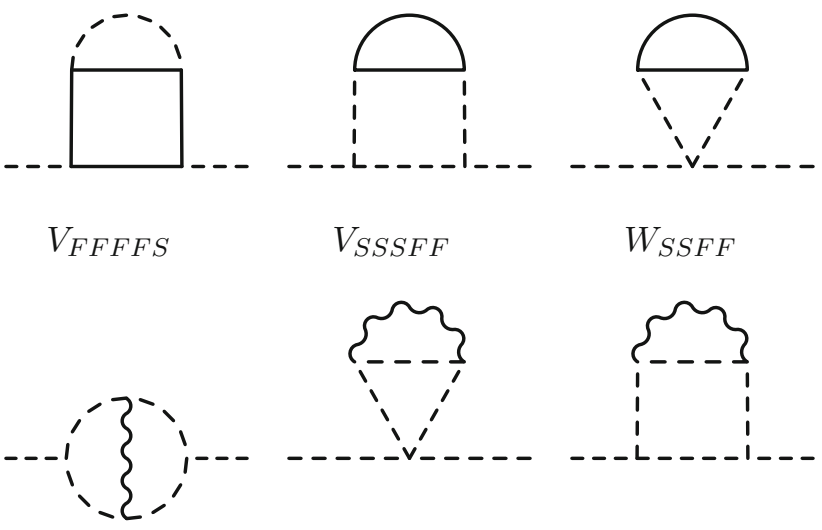

$V_{S S S F F}$

$W_{S S F F}$

$M_{S S S S V}$
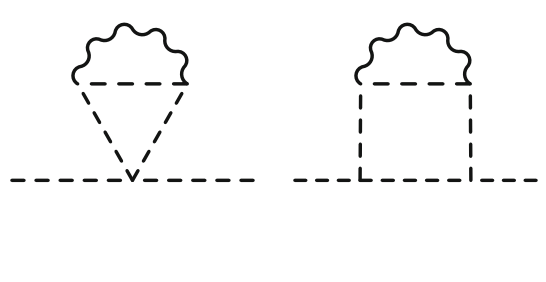

$W_{S S S V}$

$V_{S S S S V}$

Fig. 4 Remaining two-loop self-energy diagrams which do not vanish in the gaugeless limit

\subsection{Method}

For any given supersymmetric, ${ }^{2}$ model \$MODEL once the user has specified the particle content and their symmetries, SARAH calculates all of the vertices and masses. It then writes a Fortran code (placed in the suggestively named 2LPole_\$MODEL.f90) which implements our expressions, linking to a static Fortran code (named 2LPoleFunctions.f90) of the basis functions for the

$\overline{2}$ A two-loop Higgs mass calculation in non-SUSY models will become available in future releases. 
generic first and second derivatives of the effective potential defined in Sect. 2 and the appendix. These two pieces of Fortran code are called by SPheno during the calculation of the loop corrections to the Higgs mass. Here we shall give a few details of how SARAH writes 2LPole_\$MODEL. f 90. The overall algorithm is to:

1. Generate masses and couplings for all relevant particles in the gaugeless limit.

2. Populate and classify all tadpole topologies according to particle content.

3. For each tadpole topology, pass the set of diagrams along with information specifying the symmetries to a generic writer function.

4. Rotate the total tadpole vector by the Higgs rotation matrix to the non-diagonal basis, cf. Eq. (2.30).

5. Populate and classify all second derivative topologies according to particle content.

6. For each mass topology, pass the set of diagrams along with information specifying the symmetries to a generic writer function.

7. Rotate the mass matrix to the non-diagonal basis (as with the tadpoles).

The writer function is actually identical for tadpoles and mass diagrams with a switch to adjust the number of Higgses. It cycles through the list of diagrams and applies the following process for each:

(a) Determine symmetry factor of diagram due to permutations.

(b) Determine the colour factor; for diagrams with a gluon propagator this is simply $d(I) C(I)$ whereas otherwise we must trace over colour indices of the vertices. In principle, for four-point vertices there can be two colour structures for the vertex which superficially leads to more than one colour factor for such diagrams. However, as we can simply see by inspecting the expressions in the appendix, or by considering that the colour factors have to be inherited from a corresponding vacuum diagram (since differentiating with respect to neutral Higgs fields cannot introduce any additional colour factor), for diagrams with a four-point vertex consisting of four coloured fields the colour factor is given by a trace over the indices in pairs. Hence such four-point vertices are saved with the colour factor of the pairs of indices traced over. To be more explicit, such vertices can only contribute if they come from differentiating $V_{S S}^{(2)}$ which contains the coupling $\lambda^{i i j j}$. We then must simply take care that the indices of the vertices correspond correctly to the indices that are traced over.

(c) Write a nested set of loops to sum the diagram over the generations of all particles and, for the inner loop, the external Higgs legs, since the most computationally demanding aspect is evaluating the loop functions and this can be evaluated before calling the Higgs loopindeed, we also check that the coupling multiplying it is non-zero first too.

There are subtleties in translating our results into a form usable by SARAH, both stemming from the fact that in the SPheno code the couplings are stored in terms of either real or complex scalars, and four-component spinor fermions, while, since our results are based on those of Refs. $[55,56]$ and for economy we use real scalars and twocomponent fermions. The translation between the two bases as required by SARAH and SPheno is largely as described in Ref. [61]; however, here we have the additional complication for fermions of translating chains of couplings and masses such as

$C_{1}=\operatorname{Re}\left(y^{I J r} y^{K L m} y^{M N m} M_{I K}^{*} M_{J M}^{*} M_{L N}^{*}\right)$.

In SARAH, the interactions of Weyl spinors $\psi$ are derived from the corresponding Dirac spinor interactions $\Psi$ as

$$
\begin{aligned}
\text { Vertex } & =i \frac{\delta \mathcal{L}}{\delta \phi_{r} \delta \bar{\Psi}_{I} \delta \Psi_{J}} \\
& =i\left(\frac{\delta \mathcal{L}}{\delta \phi_{r} \delta \psi_{I}^{R} \delta \psi_{J}^{L}} P_{L}+\frac{\delta \mathcal{L}}{\delta \phi_{r} \delta \psi_{I}^{L *} \delta \psi_{J}^{R *}} P_{R}\right) \\
& \equiv i\left(c_{L} P_{L}+c_{R} P_{R}\right),
\end{aligned}
$$

with $\psi_{X}^{Y}=P_{Y} \Psi_{X}(Y=L, R ; X=I, J)$ and polarisation operators $P_{L}, P_{R}$; and so $c_{L, R} \leftrightarrow-y^{I J r}, c_{L, R}^{*} \leftrightarrow-y_{I J r}$. When we have complex scalars $\Phi$, we should write

$$
\begin{aligned}
\mathcal{L} \supset & \Phi^{m}\left(\bar{\Psi}_{I}\left(c_{L}(I, J, m) P_{L}+c_{R}(I, J, m) P_{R}\right) \Psi_{J}\right. \\
& +\Phi_{m}\left(\bar{\Psi}_{I}\left(c_{L}(I, J, \bar{m}) P_{L}+c_{R}(I, J, \bar{m}) P_{R}\right) \Psi_{J}\right. \\
= & \Phi^{m}\left(\bar{\Psi}_{I}\left(c_{L}(I, J, m) P_{L}+c_{R}(I, J, m) P_{R}\right) \Psi_{J}\right. \\
& +\Phi_{m}\left(\bar{\Psi}_{J}\left(c_{R}^{*}(I, J, m) P_{L}+c_{L}^{*}(I, J, m) P_{R}\right) \Psi_{J}\right.
\end{aligned}
$$

and so $c_{L}(I, J, \bar{m})=c_{R}^{*}(J, I, m)$. For a given topology, SARAH populates the diagrams using Dirac propagators (i.e. links fermions with its conjugate) and so for the coupling $C_{1}$ above we will find sets of particles

$\left\{\bar{\Psi}_{I}, \Psi_{J}, \phi^{r}\right\},\left\{\bar{\Psi}_{N}, \Psi_{I}, \phi^{m}\right\},\left\{\bar{\Psi}_{J}, \Psi_{N}, \bar{\phi}_{m}\right\}$.

Suppose each of the fermions is a Dirac spinor with Weyl spinors $\psi_{L, R}^{I}$ etc., then to construct the coupling $C$ above we must sum over the left- and right-handed Weyl fermions (which have opposite representations of all gauge groups) and thus (noting that SPheno always internally stores the fermion masses as real positive definite) 


$$
\begin{aligned}
C_{1}= & \operatorname{Re}\left[y^{\psi_{L}^{I}, \psi_{R}^{J}, r} y^{\psi_{L}^{N}, \psi_{R}^{I}, m} y_{L}^{J}, \psi_{R}^{N}, \bar{m}\right. \\
& \left.+y_{R} \psi_{R}^{I}, \psi_{L}^{J}, r y \psi_{R}^{N}, \psi_{L}^{I}, \bar{m} y \psi_{R}^{J}, \psi_{L}^{N}, m\right] M_{I} M_{J} M_{L} \\
= & -\operatorname{Re}\left[c_{R}^{*}(I, J, r) c_{R}^{*}(N, I, \bar{m}) c_{R}^{*}(J, N, m)\right. \\
& \left.+c_{L}(I, J, r) c_{L}(N, I, \bar{m}) c_{L}(J, N, m)\right] M_{I} M_{J} M_{N} \\
= & -\operatorname{Re}\left[c_{R}(I, J, r) c_{R}(N, I, \bar{m}) c_{R}(J, N, m)\right. \\
& \left.+c_{L}(I, J, r) c_{L}(N, I, \bar{m}) c_{L}(J, N, m)\right] M_{I} M_{J} M_{L}
\end{aligned}
$$

On the other hand, if the fermions are all Majorana then $\psi_{L}^{I}=\psi_{R}^{I}$ and we therefore only have half of this sum, so we include an extra factor of $1 / 2$. If we consider another example,

$C_{2}=\operatorname{Re}\left(y^{I J r} y_{I K m} y^{K N m} M_{J N}^{*}\right)$,

in SARAH we would generate the set of particles

$$
\left\{\bar{\Psi}_{I}, \Psi_{J}, \phi^{r}\right\},\left\{\bar{\Psi}_{K}, \Psi_{I}, \bar{\phi}_{m}\right\},\left\{\bar{\Psi}_{J}, \Psi_{K}, \phi^{m}\right\}
$$

and thus

$$
\begin{aligned}
C_{2} \rightarrow & \operatorname{Re}\left[y^{\psi_{L}^{I}, \psi_{R}^{J}, r} y_{\psi_{L}^{I}, \psi_{R}^{K}, \bar{m}} y^{\psi_{R}^{K}, \psi_{L}^{N}, m}\right. \\
& \left.+y^{\psi_{R}^{I}, \psi_{L}^{J}, r} y_{\psi_{R}^{I}, \psi_{L}^{K}}^{m} y_{\bar{m}}^{K}, \psi_{R}^{N}\right] M_{J} \\
\rightarrow & -\operatorname{Re}\left[c_{R}^{*}(I, J, r) c_{L}^{*}(K, I, m) c_{R}^{*}(J, K, m)\right. \\
& \left.+c_{L}(I, J, r) c_{R}(K, I, m) c_{L}(J, K, m)\right] M_{J} \\
= & -\operatorname{Re}\left[c_{R}(I, J, r) c_{L}(K, I, m) c_{R}(J, K, m)\right. \\
& \left.+c_{L}(I, J, r) c_{R}(K, I, m) c_{L}(J, K, m)\right] M_{J} .
\end{aligned}
$$

The above show that it is straightforward to translate the twocomponent results into expressions in SARAH

$$
\begin{aligned}
\operatorname{Re} & {\left[\left[\prod_{i=1}^{m} y^{I_{i} J_{i} s_{i}} \prod_{j=1}^{n} y_{I_{j}} J_{j} s_{j}\right]\left[\prod_{k=1}^{p} M_{I_{k} J_{k}}\right]\right] } \\
\rightarrow & \left(\frac{1}{2}\right)^{M}(-1)^{m+n} \operatorname{Re}\left[\prod_{i=1}^{m} c_{L}\left(I_{i}, J_{i}, s_{i}\right) \prod_{j=1}^{n} c_{R}\left(I_{j}, J_{j}, s_{j}\right)\right. \\
& \left.+\prod_{i=1}^{m} c_{R}\left(I_{i}, J_{i}, s_{i}\right) \prod_{j=1}^{n} c_{L}\left(I_{j}, J_{j}, s_{j}\right)\right]\left[\prod_{k=1}^{p} M_{I_{k}}\right]
\end{aligned}
$$

where $M=1$ for Majorana fermions and zero otherwise.

A final point regarding the translation into a basis of real and complex scalars is that the new routines assume that there is a unique way of constructing a gauge- and global symmetry-invariant coupling $\lambda^{i j k}$ from complex scalars other than the complex conjugate of the whole coupling; i.e. if $\lambda^{i j k}$ is permitted for given complex $i, j, k$ then $\lambda_{k}^{i j}$ is not. This is evidently true-if both are permitted then we can generate a holomorphic mass term at one loop which violates the premise. However, it is important in that we cannot write gauge singlets as complex scalars if they have couplings violating the above condition, no matter how small the couplings-for example for sneutrinos in see-saw models.

\subsection{How to use the new routines}

To study a model with SARAH the general procedure is as follows: the user should download and run SARAH with the required model. SARAH derives all analytical expressions for mass matrices, vertices, renormalisation group equations and loop corrections and exports this information into Fortran source code. The Fort ran source code is compiled together with SPheno and all numerical calculations are then performed by the new SPheno module. This includes a calculation of the entire mass spectrum, branching ratios as well as flavour and other precision observables [64]. For the mass spectrum all one-loop corrections to any particle are included in a diagrammatic way [21]. For a supersymmetric model there are now in addition three options to get two-loop corrections in the Higgs sector. The first two are based on the effective potential approach presented in Ref. [61], while the new routines are called by the (now default) third option.

A step-by-step description to obtain a spectrum generator for an arbitrary model \$MODEL implemented in SARAH reads as follows:

1. Download the most recent SARAH and SPheno versions into a directory $\$ \mathrm{PATH}$. Both packages are located at HepForge:

http://sarah.hepforge.org/

http://spheno.hepforge.org/

2. Enter the directory and extract both codes

$$
\begin{aligned}
& >\text { cd \$PATH } \\
& >\text { tar-xf SARAH-4.5.0.tar.gz } \\
& >\text { tar-xf SPheno-3.3.6.tar.gz }
\end{aligned}
$$

3. Start Mathematica, load SARAH, run \$MODEL, and generated the SPheno output

$<$ \$PATH/SARAH-4.5.0/SARAH.m;

Start [ "\$MODEL"] ;

MakeSPheno [ ] ;

4. Leave Mathematica, enter the SPheno directory and create a new sub-directory for your model

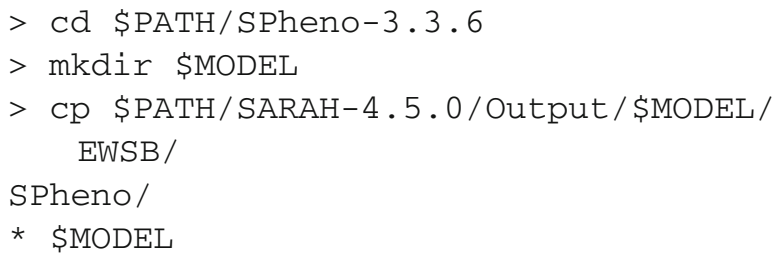

5. Compile SPheno together with the new module 
$>$ make Model $=\$$ MODEL

After these steps a new binary bin/SPheno\$MODEL is available. To run it an input file in the Les Houches format is needed. SARAH writes a template for that file which has to be filled with numbers. To enable the new functions for a calculation of the two-loop Higgs masses based on our new loop functions the following flags have to be set:

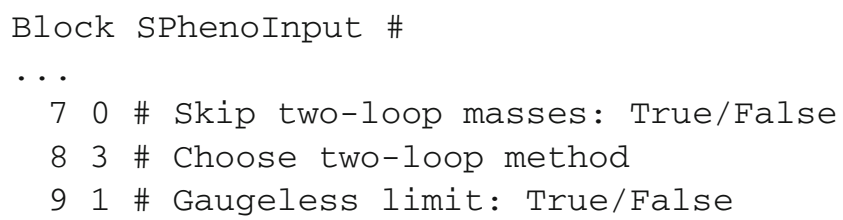

$8->3$ chooses the new approach to calculate the loop corrections. The other options for flag 8 would correspond the effective potential calculations based on SARAH $(8->1$ for a fully numerical derivation, $8->2$ for a semi-analytical derivation). Also some hard-coded corrections are available which are based on results in the literature: 8->8 uses the known $\alpha_{S}\left(\alpha_{b}+\alpha_{t}\right)$ corrections for the MSSM, NMSSM, TMSSM or any variant thereof with up to four neutral CPeven Higgs fields and including models with Dirac gauginos [65]. 8->9 uses the corrections of option 8 and adds the two-loop MSSM $\left(\alpha_{t}+\alpha_{b}+\alpha_{\tau}\right)^{2}$ results based on Refs. [35$37,40,41]$. Note that the last two options are not included by default in the SPheno output of SARAH. To include them, the user must make sure to include in the SPheno.m of the considered model

Use2LoopFromLiterature = True;
Finally, SPheno is executed by

$>$. / bin/SPheno\$MODEL \$MODEL/LesHouches. in.

\$MODEL

and the output is written to

SPheno.spc. \$MODEL

\subsection{Validation}

We have intensively used the SPheno output to validate our new two-loop functions, in particular:

- We found a numerical agreement of more than 10 digits between our code and using public routines for the MSSM based on Refs. [35-37,40,41] for the selfenergies. In order to perform this validation, it is necessary to use the same assumptions: turn off the first and second generation Yukawa couplings; take the Goldstone boson and light Higgs masses in the loops to be zero, and set the tree-level mixing angle of the neutral CP-even scalars to $\alpha=\beta-\pi / 2$.

The excellent agreement between all four possibilities to calculate the two-loop Higgs masses in the CMSSM is also shown in Fig. 5. The parameter point used here is the same as in [61],

$$
\begin{aligned}
M_{0} & =M_{1 / 2}=1 \mathrm{TeV}, \quad A_{0}=-2 \mathrm{TeV}, \\
\tan \beta & =10, \quad \operatorname{sgn}(\mu)=1 .
\end{aligned}
$$

Fig. 5 Comparison between the diagrammatic calculation of the two-loop Higgs masses with SARAH/SPheno presented here (diag: diagrammatical) with the effective potential calculation of Ref. [61] (p-num purely numerical, semi semi-analytical), and with the routines based on

Refs. [35-37,40,41] (ref reference). The fixed parameters are those in Eq. (3.9)
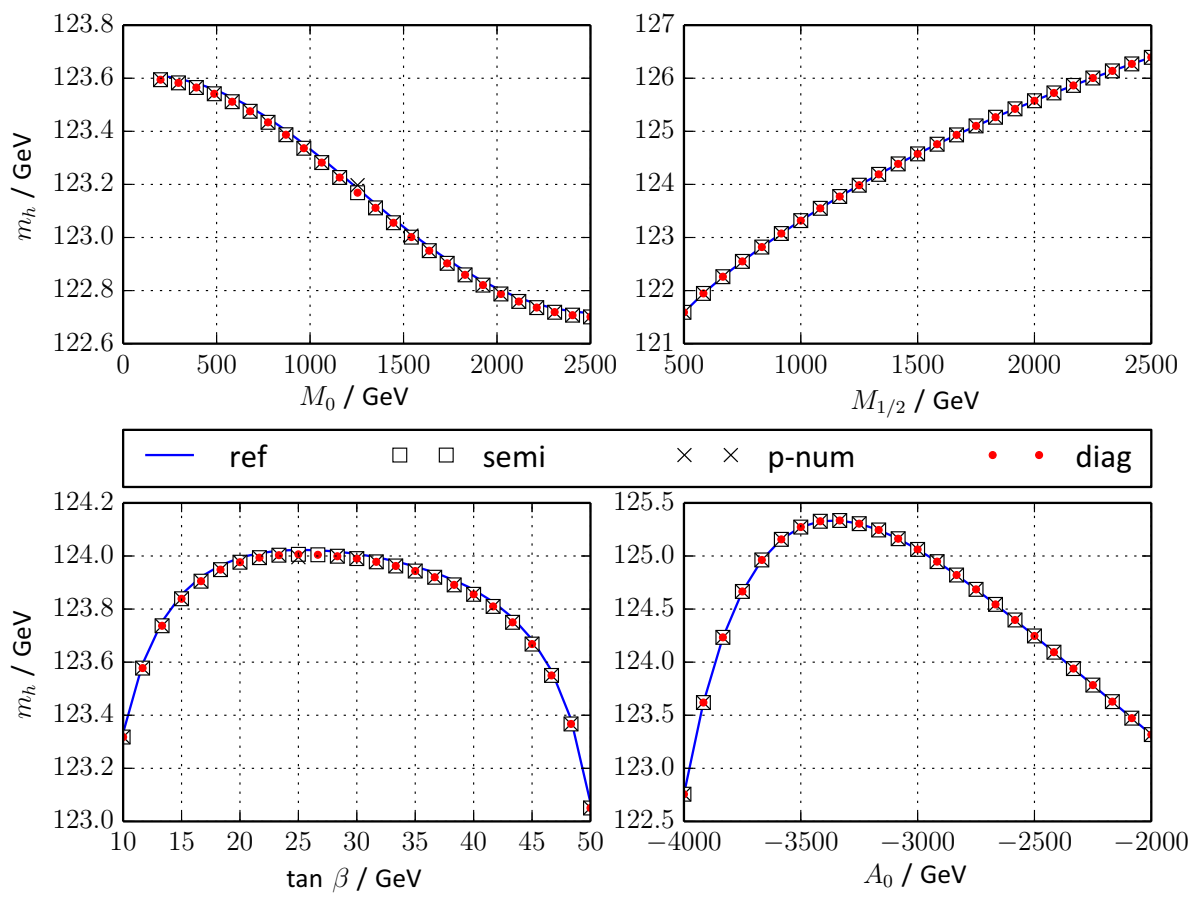
- Similarly, we found full agreement with available results for the $\alpha_{s}\left(\alpha_{b}+\alpha_{t}\right)$ corrections in the NMSSM [20] and for Dirac gauginos [65].

- We compared the full two-loop corrections, i.e. also including corrections not involving the strong interaction, for the NMSSM, models with Dirac gauginos as well as for the B-L-SSM [66] against the results using the other two options based on a completely independent implementation in SARAH presented in Ref. [61]. We usually found very good agreement. Tiny differences were based on numerical artefacts in the routines using the effective potential ansatz. Similarly, we could reproduce the results of Ref. [67] for the two-loop contributions to the Higgs mass stemming from $R$-parity violating couplings.

The new routines of course provide better stability. For example, in the routines based on numerically taking the derivatives of the potential, it is necessary to take care with the initial step size; if there are neutral scalars which have small expectation values then the results from those methods could become inaccurate- this problem occurs in general for any neutral scalar having expectation value $v_{i} \ll M_{\text {SUSY }}$. Less significantly, the numerical method can suffer from (small) errors when there are small couplings present, such that they do not induce a sufficient shift in particle masses or couplings upon variation of the Higgs vevs to accurately take the derivative. Hence, it is very important to have two independent implementations of generic two-loop Higgs mass calculations in SARAH/SPheno: this is the only possibility to cross check results for models beyond the (N)MSSM at the moment. Thus, we highly urge users to test all options for the model under consideration and to compare the results.

\section{Summary}

We have presented the derivation of a new set of expressions for calculation of the tadpoles and self-energies at the twoloop level. These expressions include all generic diagrams which do not vanish in the gaugeless limit and are valid in the limit of zero external momenta. This set of loop functions is simpler than the set of expressions obtained by taking the limit $p^{2} \rightarrow 0$ in the pole mass functions available in the literature so far. This allows for a rapid numerical evaluation of the Higgs mass. We have implemented these functions in Fortran and included them in the new version of SARAH 4.5 .0 . This provides the possibility to automatically calculate the Higgs mass in a wide range of supersymmetric models with a guaranteed numerical accuracy and stability. The obtained precision for the Higgs mass is comparable with the one dedicated spectrum generators have provided so far for the MSSM, and they can now be applied to the study of a wide variety of models.
Aside from accuracy and stability, one of the principal advantages of this approach is that it is readily extendable. It would be straightforward to extend the calculation to nonzero momentum by changing the functions in the code and linking with the library TSIL. On the other hand, including the electroweak contributions should be possible by applying these techniques to the full effective potential; we presented the expressions in this case for the tadpoles in the appendix, but the second derivatives are currently unknown-as are the full set of equivalent expressions in the diagrammatic approach. Furthermore, to truly reach the full two-loop precision we would require the two-loop shift in the $Z$-mass that determines the electroweak expectation value. We hope to return to these issues in the future.

Acknowledgments We are indebted to Pietro Slavich for a large number of fruitful discussions, and comments on the draft.

Open Access This article is distributed under the terms of the Creative Commons Attribution 4.0 International License (http://creativecomm ons.org/licenses/by/4.0/), which permits unrestricted use, distribution, and reproduction in any medium, provided you give appropriate credit to the original author(s) and the source, provide a link to the Creative Commons license, and indicate if changes were made. Funded by $\mathrm{SCOAP}^{3}$.

\section{Appendix A: Loop functions}

The pole masses are constructed from various one- and twoloop functions which are defined in Ref. [57]. However, for the purposes of calculating the effective potential these can be reduced to combinations of standard expressions involving just the usual functions $I$ and $J$. Here we compile this dictionary. We stick closely to the notation of Ref. [57] and make often use of results presented there. Note that we use the standard notation

$\overline{\ln } x \equiv \log \frac{x}{Q^{2}}$

where $Q$ is the renormalisation scale.

\section{A.1: One-loop functions}

At the one-loop level only two functions are needed:

$$
\begin{aligned}
A(x) & \equiv \lim _{\epsilon \rightarrow 0}[\mathbf{A}(x)+x / \epsilon]=x(\overline{\ln } x-1), \\
B(x, y) & \equiv \lim _{\epsilon \rightarrow 0}[\mathbf{B}(x, y)-1 / \epsilon] \\
& =-\int_{0}^{1} \mathrm{~d} t \overline{\ln }[t x+(1-t) y-t(1-t) s]
\end{aligned}
$$


Clearly, we find the following relation to $J(x)$, which is widely used at two loops (recall $C \equiv 16 \pi^{2} \mu^{2 \epsilon}(2 \pi)^{-d}$ ):

$$
\begin{aligned}
& A(x)=J(x), \quad \mathbf{A}(x)=\mathbf{J}(x)=J(x)-\frac{x}{\epsilon}, \\
& \mathbf{B}(x, y) \underset{p^{2} \rightarrow 0}{\longrightarrow} C \int d^{d} k \frac{1}{\left(k^{2}+x\right)\left(k^{2}+y\right)}=\frac{1}{y-x}(\mathbf{J}(x)-\mathbf{J}(y)), \\
& B(x, y) \underset{p^{2} \rightarrow 0}{\longrightarrow} \frac{1}{y-x}(J(x)-J(y)) \equiv B_{0}(x, y),
\end{aligned}
$$

where we have introduced the subscript to denote that the external momentum is zero. Let us also denote for future use

$C_{0}(x, y, z) \equiv \frac{B_{0}(x, z)-B_{0}(x, y)}{y-z}$,

which is actually symmetric on all three indices.

\section{A.2: Two-loop functions}

At the two-loop level we make use of the following set of functions:

$$
\begin{aligned}
J(x, y) & \equiv J(x) J(y), \\
S_{0}(x, y, z) & =I(x, y, z), \\
T_{0}(x, y, z) & =-\frac{\partial}{\partial x} I(x, y, z), \\
U_{0}(x, y, z, u) & =\frac{1}{y-x}(I(x, z, u)-I(y, z, u)), \\
V_{0}(x, y, z, u) & =-\frac{\partial}{\partial y} U_{0}(x, y, z, u), \\
\bar{T}_{0}(x, y) & =\lim _{\delta \rightarrow 0}\left[T_{0}(\delta, x, y)+B_{0}(x, y) \overline{\ln } \delta\right]
\end{aligned}
$$

together with

$$
\begin{aligned}
& \mathbf{M}(x, y, z, u, v) \rightarrow C^{2} \int d^{d} k \\
& \quad \times \int d^{d} q \frac{1}{\left(k^{2}+x\right)\left(q^{2}+y\right)\left(k^{2}+z\right)\left(q^{2}+u\right)\left((k-q)^{2}+v\right)} \\
& =\frac{1}{(u-y)}(\mathbf{U}(x, z, y, v)-\mathbf{U}(x, z, u, v)), \\
& M_{0}(x, y, z, u, v)=\frac{1}{(u-y)}\left(U_{0}(x, z, y, v)-U_{0}(x, z, u, v)\right) .
\end{aligned}
$$

These functions have to following properties: (i) $I(x, y, z)$ is symmetric in all arguments; (ii) $T_{0}(x, y, z)$ is symmetric in the last two arguments; (iii) $U_{0}(x, y, z, u)$ is invariant under the exchange $z \leftrightarrow u$ and $x \leftarrow y$; (iv) $M_{0}(x, y, z, u, v)$ is invariant under the interchanges $(x, z) \leftrightarrow(y, u),(x, y) \leftrightarrow$ $(z, u)$, and $(x, y) \leftrightarrow(u, z)$.
An explicit expression for $I(x, y, z)$ is for instance given by [55]

$$
\begin{aligned}
& I(x, y, z)=\frac{1}{2}(x-y-z) \overline{\ln } y \overline{\ln } z+\frac{1}{2}(y-x-z) \overline{\ln } x \overline{\ln } z \\
& \quad+\frac{1}{2}(z-x-y) \overline{\ln } x \overline{\ln } y \\
& \quad+2 x \overline{\ln } x+2 y \overline{\ln } y+2 z \overline{\ln } z-\frac{5}{2}(x+y+z) \\
& \quad-\frac{1}{2} \xi(x, y, z)
\end{aligned}
$$

with

$$
\begin{aligned}
& \xi(x, y, z)=R[2 \ln [(z+x-y-R) / 2 z] \ln \\
& \quad \times[(z+y-x-R) / 2 z]-\ln \frac{x}{z} \ln \frac{y}{z} \\
& \quad-2 \operatorname{Li}_{2}[(z+x-y-R) / 2 z] \\
& \left.\quad-2 \operatorname{Li}_{2}[(z+y-x-R) / 2 z]+\frac{\pi^{2}}{3}\right], \\
& R=\sqrt{x^{2}+y^{2}+z^{2}-2 x y-2 x z-2 y z .}
\end{aligned}
$$

Note that $x, y \leq z$ has been assumed here.

\section{A.3: Relations required for the pole functions}

The above loop functions are used as a basis for the various loop functions. However, we also find additional combinations such as

$$
\begin{aligned}
V_{S S S S S}(x, y, z, u, v)= & \frac{1}{y-z}\left(U_{0}(x, y, u, v)-U_{0}(x, z, u, v)\right) \\
& \sim-\frac{1}{\left(k^{2}+x\right)\left(k^{2}+y\right)\left(k^{2}+z\right)} \\
& \times \frac{1}{\left(q^{2}+u\right)\left((q+k)^{2}+v\right)} .
\end{aligned}
$$

We should compare this to

$$
V(x, y, z, u) \sim \frac{1}{\left(k^{2}+x\right)\left(k^{2}+y\right)^{2}} \frac{1}{\left(q^{2}+u\right)\left((q+k)^{2}+v\right)} .
$$

Hence we can write $V_{S S S S}(x, y, y, u, v)=-V(x, y, z, u)$ and remember that $V_{S S S S S}$ is symmetric on its first three entries.

\section{A.4: Simplified loop functions}

For the amplitudes with one or more massless (and possibly identical) fields we find simplified expressions for the loop integrals, some of which are collected below. 


$$
\begin{aligned}
I(x, y, 0)= & \frac{1}{2}\left(-5 x-5 y+(-x+y) \overline{\ln }^{2} x+4 y \overline{\ln } y\right. \\
& \left.+\overline{\ln } x(4 x-2 y \overline{\ln } y)-2(x-y) \operatorname{Li}_{2}(1-y / x)\right),
\end{aligned}
$$

$$
\begin{aligned}
I(x, x, 0) & =x\left(-5+4 \overline{\ln } x-\overline{\ln }^{2} x\right) \\
I(x, 0,0) & =-\frac{1}{2} x(\overline{\ln } x)^{2}+2 x \overline{\ln } x-\frac{5}{2} x-\frac{\pi^{2}}{6} x \\
B_{0}(x, x) & =-\frac{\ln x}{}
\end{aligned}
$$

It is also sometimes necessary to consider the case of small mass splittings. The results for $I(\delta, x, y), I(\delta, x, x)$, $I\left(\delta, \delta^{\prime}, x\right)$ in the limits $\delta \rightarrow 0, \delta^{\prime} \rightarrow 0$ can be found in Ref. [55] and we do not repeat them here.

\section{Appendix B: Second derivatives of the effective potential}

In this appendix we present the results for the second derivatives of the effective potential. Largely these are identical to those in [56] with the external momentum set to zero, but for the sake of completeness we repeat the full set here. However, certain expressions become much simpler in this limit, notably some complicated functions involving both fermion and scalar propagators, and those involving gauge bosons.

The full contribution is

$$
\begin{aligned}
\Pi_{i j}= & \Pi_{i j}^{S}+\Pi_{i j}^{S F(W)}+\Pi_{i j}^{S F_{4}(M)}+\Pi_{i j}^{S_{2} F_{3}(M)} \\
& +\Pi_{i j}^{S_{3} F_{2}(V)}+\Pi_{i j}^{S F_{4}(V)}+\Pi_{i j}^{S V}+\Pi_{i j}^{F V} .
\end{aligned}
$$

\section{B.1: Diagrams with only scalar propagators}

The first contribution, including only scalar propagators, comprises the eight diagrams shown in Fig. 3. These are unchanged from [56] and are given by

$$
\begin{aligned}
\Pi_{i j}^{S}= & \frac{1}{4} \lambda^{i j k l} \lambda^{k m n} \lambda^{l m n} W_{S S S S}\left(m_{k}^{2}, m_{l}^{2}, m_{m}^{2}, m_{n}^{2}\right) \\
& +\frac{1}{4} \lambda^{i j k l} \lambda^{k l m m} X_{S S S}\left(m_{k}^{2}, m_{l}^{2}, m_{m}^{2}\right) \\
& +\frac{1}{2} \lambda^{i k l} \lambda^{j k m} \lambda^{l m n n} Y_{S S S S}\left(m_{k}^{2}, m_{l}^{2}, m_{m}^{2}, m_{n}^{2}\right) \\
& +\frac{1}{4} \lambda^{i k l} \lambda^{j m n} \lambda^{k l m n} Z_{S S S S}\left(m_{k}^{2}, m_{l}^{2}, m_{m}^{2}, m_{n}^{2}\right) \\
& +\frac{1}{6} \lambda^{i k l m} \lambda^{j k l m} S_{S S S}\left(m_{k}^{2}, m_{l}^{2}, m_{m}^{2}\right) \\
& +\frac{1}{2}\left(\lambda^{i k l} \lambda^{j k m n}+\lambda^{j k l} \lambda^{i k m n}\right) \\
& \times \lambda^{l m n} U_{S S S S}\left(m_{k}^{2}, m_{l}^{2}, m_{m}^{2}, m_{n}^{2}\right) \\
& +\frac{1}{2} \lambda^{i k l} \lambda^{j k m} \lambda^{l n p} \lambda^{m n p} V_{S S S S S}\left(m_{k}^{2}, m_{l}^{2}, m_{m}^{2}, m_{n}^{2}, m_{p}^{2}\right)
\end{aligned}
$$

$$
+\frac{1}{2} \lambda^{i k m} \lambda^{j l n} \lambda^{k l p} \lambda^{m n p} M_{S S S S S}\left(m_{k}^{2}, m_{l}^{2}, m_{m}^{2}, m_{n}^{2}, m_{p}^{2}\right)
$$

Here the loop integral functions are given by

$$
\begin{aligned}
W_{S S S S}(x, y, z, u) & =U_{0}(x, y, z, u), \\
X_{S S S}(x, y, z) & =-J(z) B_{0}(x, y), \\
Y_{S S S S}(x, y, z, u) & =J(u) C_{0}(x, y, z), \\
Z_{S S S S}(x, y, z, u) & =B_{0}(x, y) B_{0}(z, u), \\
S_{S S S}(x, y, z)= & -I(x, y, z), \\
U_{S S S S}(x, y, z, u)= & U_{0}(x, y, z, u), \\
V_{S S S S S}(x, y, z, u, v)= & {\left[U_{0}(x, y, u, v)\right.} \\
& \left.-U_{0}(x, z, u, v)\right] /(y-z), \\
M_{S S S S S}(x, y, z, u, v)= & -M_{0}(x, y, z, u, v) .
\end{aligned}
$$

In the case that $y=z$, we have the simplification

$V_{S S S S}(x, y, y, u, v)=-V(x, y, u, v)$.

It should be noted (for example, for the purposes of evaluating the colour factors) that topologies $X_{S S S}, Y_{S S S}, Z_{S S S}$ arise from differentiating $V_{S S}^{(2)}$ (and hence the tadpole $T_{S S}$ ) while the others arise from differentiating $V_{S S S}^{(2)}$ (and hence the tadpoles $T_{S S S}$ and $T_{S S S S}$.

B.2: Diagrams with scalar and fermion propagators

The contributions from diagrams with the topology $W$ are

$$
\begin{aligned}
\Pi_{i j}^{S F(W)}= & \frac{1}{2} \lambda^{i j k l} \operatorname{Re}\left[y^{M N k} y^{M^{\prime} N^{\prime} l} M_{M M^{\prime}} M_{N N^{\prime}}\right] \\
& \times W_{S S \overline{F F}}\left(m_{k}^{2}, m_{l}^{2}, m_{M}^{2}, m_{N}^{2}\right) \\
& +\frac{1}{2} \lambda^{i j k l} y^{M N k} y_{M N l} \\
& \times W_{S S F F}\left(m_{k}^{2}, m_{l}^{2}, m_{M}^{2}, m_{N}^{2}\right)
\end{aligned}
$$

where we can slightly simplify the loop functions:

$$
\begin{aligned}
W_{S S F F}(x, y, z, u)= & -2 W_{S S S S}(x, y, z, u), \\
W_{S S F F}(x, y, z, u)= & -(z+u-y) U_{0}(x, y, z, u) \\
& -I(x, z, u)+B_{0}(x, y)(J(z)+J(u)) .
\end{aligned}
$$

The contributions from the diagrams of the topology $M$ with four fermions are

$$
\begin{aligned}
\Pi_{i j}^{S F_{4}(M)}= & \operatorname{Re}\left[y^{K M i} y^{L N j} y^{K^{\prime} L^{\prime} p} y^{M^{\prime} N^{\prime} p}\right. \\
& \left.\times M_{K K^{\prime}} M_{L L^{\prime}} M_{M M^{\prime}} M_{N N^{\prime}}\right] \\
& \times M_{\overline{F F F F} S}\left(m_{K}^{2}, m_{L}^{2}, m_{M}^{2}, m_{N}^{2}, m_{p}^{2}\right)
\end{aligned}
$$




$$
\begin{aligned}
& +2 \operatorname{Re}\left[y^{K M i} y_{L N j} y_{K L^{\prime} p} y^{M^{\prime} N p} M^{L L^{\prime}} M_{M M^{\prime}}\right] \\
& \times M_{F \overline{F F} F}\left(m_{K}^{2}, m_{L}^{2}, m_{M}^{2}, m_{N}^{2}, m_{p}^{2}\right) \\
& +\operatorname{Re}\left[\left(y^{K M i} y_{L N j}+y^{K M j} y_{L N i}\right)\right. \\
& \left.\times y_{K L^{\prime} p} y_{M N^{\prime} p} M^{L L^{\prime}} M^{N N^{\prime}}\right] \\
& \times M_{F \bar{F} F \bar{F} S}\left(m_{K}^{2}, m_{L}^{2}, m_{M}^{2}, m_{N}^{2}, m_{p}^{2}\right) \\
& +2 \operatorname{Re}\left[y^{K M i} y^{L N j} y_{K L p} y^{M^{\prime} N^{\prime} p} M_{M M^{\prime}} M_{N N^{\prime}}\right] \\
& \times M_{F F \overline{F F} S}\left(m_{K}^{2}, m_{L}^{2}, m_{M}^{2}, m_{N}^{2}, m_{p}^{2}\right) \\
& +\operatorname{Re}\left[y^{K M i} y^{L N j} y_{K L p} y_{M N p}\right] \\
& \times M_{F F F F S}\left(m_{K}^{2}, m_{L}^{2}, m_{M}^{2}, m_{N}^{2}, m_{p}^{2}\right),
\end{aligned}
$$

where

$$
\begin{aligned}
& M_{\overline{F F F F} S}(x, y, z, u, v)=2 M_{0}(x, y, z, u, v), \\
& M_{F \overline{F F} S}(x, y, z, u, v)=(y+z-v) M_{0}(x, y, z, u, v) \\
& \quad-U_{0}(x, z, u, v)-U_{0}(u, y, x, v) \\
& \quad+B_{0}(x, z) B_{0}(y, u), \\
& M_{F \bar{F} F \bar{F} S}(x, y, z, u, v)=(x+z) M_{0}(x, y, z, u, v) \\
& \quad-U_{0}(y, u, z, v)-U_{0}(u, y, x, v), \\
& M_{F F \overline{F F} S}(x, y, z, u, v)=(x+y-v) M_{0}(x, y, z, u, v) \\
& \quad-U_{0}(x, z, u, v)-U_{0}(y, u, z, v) \\
& \quad+B_{0}(x, z) B_{0}(y, u) \\
& M_{F} F F S(x, y, z, u, v)=(x u+y z) M_{0}(x, y, z, u, v) \\
& \quad-x U_{0}(z, x, y, v)-z U_{0}(x, z, u, v) \\
& \quad-u U_{0}(y, u, z, v)-y U_{0}(u, y, x, v) \\
& \quad+I(x, u, v)+I(y, z, v) .
\end{aligned}
$$

The results from the diagrams of the topology $M$ with three fermions are

$$
\begin{aligned}
\Pi_{i j}^{S_{2} F_{3}(M)}= & \lambda^{i k m}\left(\operatorname{Re}\left[y^{L N j} y^{L^{\prime} P k} y^{N^{\prime} P^{\prime} m} M_{L L^{\prime}} M_{N N^{\prime}} M_{P P^{\prime}}\right]\right. \\
& \times M_{S \bar{F} S \overline{F F}}\left(m_{k}^{2}, m_{L}^{2}, m_{m}^{2}, m_{N}^{2}, m_{P}^{2}\right) \\
& +2 \operatorname{Re}\left[y^{L N j} y_{L P k} y^{N^{\prime} P m} M_{N N^{\prime}}\right] \\
& \times M_{S F S \bar{F} F}\left(m_{k}^{2}, m_{L}^{2}, m_{m}^{2}, m_{N}^{2}, m_{P}^{2}\right) \\
& +\operatorname{Re}\left[y^{L N j} y_{L P k} y_{N P^{\prime} m} M^{P P^{\prime}}\right] \\
& \left.\times M_{S F S F \bar{F}}\left(m_{k}^{2}, m_{L}^{2}, m_{m}^{2}, m_{N}^{2}, m_{P}^{2}\right)\right)+(i \leftrightarrow j),
\end{aligned}
$$

where

$$
\begin{aligned}
& M_{S \bar{F} \overline{F F}}(x, y, z, u, v)=2 M_{0}(x, y, z, u, v), \\
& M_{S F S \bar{F} F}(x, y, z, u, v)=(v-x+y) M_{0}(x, y, z, u, v) \\
& \quad+U_{0}(y, u, z, v)-U_{0}(x, z, u, v) \\
& \quad-B_{0}(x, z) B_{0}(y, u) \\
& M_{S F S F \bar{F}}(x, y, z, u, v)=(y+u) M_{0}(x, y, z, u, v)
\end{aligned}
$$

$$
-U_{0}(x, z, u, v)-U_{0}(z, x, y, v) .
$$

The contributions from the diagrams of the topology $V$, with three scalars and two fermions are

$$
\begin{aligned}
& \Pi_{i j}^{S_{3} F_{2}(V)}=\lambda^{i k l} \lambda^{j k m}\left(\operatorname{Re}\left[y^{N P l} y^{N^{\prime} P^{\prime} m} M_{N N^{\prime}} M_{P P^{\prime}}\right]\right. \\
& \quad \times V_{S S S \overline{F F}}\left(m_{k}^{2}, m_{l}^{2}, m_{m}^{2}, m_{N}^{2}, m_{P}^{2}\right) \\
& \quad+\operatorname{Re}\left[y^{N P l} y_{N P m}\right] \\
& \left.\quad \times V_{S S S F F}\left(m_{k}^{2}, m_{l}^{2}, m_{m}^{2}, m_{N}^{2}, m_{P}^{2}\right)\right),
\end{aligned}
$$

where

$$
\begin{aligned}
V_{S S S \overline{F F}}(x, y, z, u, v)= & -2 V_{S S S S S}(x, y, z, u, v), \quad(\mathrm{B} .26) \\
V_{S S S F F}(x, y, z, u, v)= & U_{0}(x, y, u, v) \\
& +(z-u-v) V_{S S S S}(x, y, z, u, v) \\
& -(J(u)+J(v)) C_{0}(x, y, z) .
\end{aligned}
$$

The results from the diagrams of the topology $V$ with four fermions are

$$
\begin{aligned}
& \Pi_{i j}^{S F_{4}(V)}=2 \operatorname{Re}\left[y^{K L i} y^{K^{\prime} M j} y^{L^{\prime} N p} y^{M^{\prime} N^{\prime} p} M_{K K^{\prime}} M_{L L^{\prime}} M_{M M^{\prime}} M_{N N^{\prime}}\right] \\
& \times V_{\overline{F F F F} S}\left(m_{K}^{2}, m_{L}^{2}, m_{M}^{2}, m_{N}^{2}, m_{p}^{2}\right) \\
& +2 \operatorname{Re}\left[\left(y^{K L i} y^{K^{\prime} M j}+y^{K L j} y^{K^{\prime} M i}\right)\right. \\
& \left.\times y_{L N p} y^{M^{\prime} N p} M_{K K^{\prime}} M_{M M^{\prime}}\right] \\
& \times V_{\bar{F} F \bar{F} F S}\left(m_{K}^{2}, m_{L}^{2}, m_{M}^{2}, m_{N}^{2}, m_{p}^{2}\right) \\
& +2 \operatorname{Re}\left[y^{K L i} y^{K^{\prime} M j} y_{L N p} y_{M N^{\prime} p} M_{K K^{\prime}} M^{N N^{\prime}}\right] \\
& \times V_{\bar{F} F F \bar{F} S}\left(m_{K}^{2}, m_{L}^{2}, m_{M}^{2}, m_{N}^{2}, m_{p}^{2}\right) \\
& +2 \operatorname{Re}\left[y^{K L i} y_{K M j} y^{L^{\prime} N p} y_{M^{\prime} N p} M_{L L^{\prime}} M^{M M^{\prime}}\right] \\
& \times V_{F \overline{F F} F S}\left(m_{K}^{2}, m_{L}^{2}, m_{M}^{2}, m_{N}^{2}, m_{p}^{2}\right) \\
& +2 \operatorname{Re}\left[\left(y^{K L i} y_{K M j}+y^{K L j} y_{K M i}\right)\right. \\
& \left.\times y_{L N p} y_{M^{\prime} N^{\prime} p} M^{M M^{\prime}} M^{N N^{\prime}}\right] \\
& \times V_{F F \overline{F F} S}\left(m_{K}^{2}, m_{L}^{2}, m_{M}^{2}, m_{N}^{2}, m_{p}^{2}\right) \\
& +2 \operatorname{Re}\left[y^{K L i} y_{K M j} y_{L N p} y^{M N p}\right] \\
& \times V_{F F F F S}\left(m_{K}^{2}, m_{L}^{2}, m_{M}^{2}, m_{N}^{2}, m_{p}^{2}\right) \text {, }
\end{aligned}
$$

where

$V_{\overline{F F F F S}}(x, y, z, u, v)=-2 V_{S S S S S}(x, y, z, u, v)$,

$V_{\bar{F} F \bar{F} F S}(x, y, z, u, v)=-U_{0}(x, y, u, v)$

$+(v-z-u) V_{S S S S S}(x, y, z, u, v)$

$-(J(v)-J(u)) C_{0}(x, y, z)$,

$V_{\bar{F} F F \bar{F} S}(x, y, z, u, v)=-2 U_{0}(x, y, u, v)$

$-2 z V_{S S S S S}(x, y, z, u, v)$,

$V_{F \overline{F F} F S}(x, y, z, u, v)=f_{F F S}^{(2,0,0)}(x, y, z ; u, v)$, 


$$
\begin{aligned}
& V_{F F \overline{F F S}}(x, y, z, u, v)=-U_{0}(x, y, u, v) \\
& \quad-U_{0}(y, z, u, v)-(x+z) \\
& \quad \times V_{S S S S S}(x, y, z, u, v) \\
& \quad V_{F F F F S}(x, y, z, u, v)=f_{F F S}^{(1,0,0)}(y, z, u ; v) \\
& \quad+x f_{F F S}^{(2,0,0)}(x, y, z, u ; v) .
\end{aligned}
$$

These represent significant simplifications over the full pole contributions. To recapitulate,

$$
\begin{aligned}
& f_{F F S}^{(1,0,0)}(x, y, u ; v) \equiv B_{0}(x, y)(J(v)-J(u)) \\
& \quad+I(x, u, v)-(y+u-v) \\
& \quad \times U_{0}(x, y, u, v) \\
& f_{F F S}^{(2,0,0)}(x, y, z, u ; v) \equiv C_{0}(x, y, z)(J(u)-J(v)) \\
& \quad-U_{0}(x, z, u, v)-(y+u-v) \\
& \quad \times V_{S S S S S}(x, y, z, u, v) .
\end{aligned}
$$

Note that $f_{F F S}^{(2,0,0)}$ is symmetric on its first three indices.

\section{B.3: Diagrams with one vector propagator}

For self-energies of neutral scalars where all gauge groups are unbroken, the diagrams involving one vector propagator are particularly simple.

\section{B.3.1: Diagrams with scalars}

We have for diagrams involving scalars

$$
\begin{aligned}
\Pi_{i j}^{S V}= & \frac{1}{2} g^{2} d(i) C(i)\left[\lambda^{i j k k} W_{S S S V}\left(m_{k}^{2}, m_{k}^{2}, m_{k}^{2}, 0\right)\right. \\
& \left.+\lambda^{i k l} \lambda^{j k l} G_{S S}\left(m_{k}^{2}, m_{l}^{2}\right)\right] .
\end{aligned}
$$

In [56] the functions are given as (setting the external momentum to zero)

$$
\begin{aligned}
W_{S S S V}(x, x, x, 0) & \equiv 3 I(x, x, 0)-J(x)+2 x, \\
G_{S S}(x, y) \equiv & 4 y V(x, y, y, 0)+4 x V(y, x, x, 0) \\
& -2 U_{0}(x, y, y, 0)-2 U_{0}(y, x, x, 0)
\end{aligned}
$$

$$
\begin{aligned}
& -2 J(y) B_{0}\left(x, y^{\prime}\right)-2 J(x) B_{0}\left(y, x^{\prime}\right) \\
& +2(x+y) M(x, x, y, y, 0) \\
& -2 U_{0}(x, y, y, 0) \\
& -2 U_{0}(y, x, x, 0)+B_{0}(x, y)^{2} .
\end{aligned}
$$

However, the expression for $G_{S S}$ greatly simplifies, as we could see by taking the derivative of (2.39):

$$
\begin{aligned}
G_{S S}(x, y)= & 2\left[-U_{0}\left(m_{i}^{2}, m_{k}^{2}, m_{k}^{2}, 0\right)-U_{0}\left(m_{m}^{2}, m_{i}^{2}, m_{i}^{2}, 0\right)\right. \\
& \left.\left.+B_{0}\left(m_{i}^{2}, m_{k}^{2}\right)+2\right)\right] \\
= & -12+\frac{11(x \overline{\ln } x-y \overline{\ln } y)-3\left(x \overline{\ln }^{2} x-y \overline{\ln }^{2} y\right)}{x-y}, \\
G_{S S}(x, x)= & -1+5 \overline{\ln } x-3 \overline{\ln }^{2} x .
\end{aligned}
$$

\section{B.3.2: Diagrams with fermions}

For the diagrams involving fermions we obtain

$$
\begin{aligned}
\Pi_{i j}^{F V}= & g^{2} d(K) C(K)\left[\operatorname{Re}\left(y^{i K L} y_{j K L}\right) G_{F F}\left(m_{K}^{2}, m_{L}^{2}\right)\right. \\
& \left.+\operatorname{Re}\left(y^{i K L} y^{j K^{\prime} L^{\prime}} M_{K K^{\prime}} M_{L L^{\prime}}\right) G_{\overline{F F}}\left(m_{K}^{2}, m_{L}^{2}\right)\right] .
\end{aligned}
$$

Here we have the simpler expressions

$$
\begin{aligned}
G_{F F}(x, y) \equiv & 2(x+y)\left[3 U_{0}(x, y, x, 0)+3 U_{0}(x, y, y, 0)\right. \\
& \left.-5 B_{0}(x, y)\right]-6 I(x, x, 0)-6 I(y, y, 0) \\
& +10 J(x)+10 J(y)-16(x+y) \\
& +\delta_{\overline{M S}} 4\left[J(x)+J(y)-(x+y) B_{0}(x, y)\right] \\
G_{\overline{F F}}(x, y) \equiv & 4\left(3 U_{0}(x, y, x, 0)+3 U_{0}(x, y, y, 0)\right. \\
& \left.-5 B_{0}(x, y)-4\right) \\
& -\delta_{\overline{M S}} 4\left[1+2 B_{0}(x, y)\right] .
\end{aligned}
$$

\section{Appendix C: Derivatives of effective potential with massive vectors}

In this appendix we shall present the full results for the tadpoles including the possibility of massive gauge bosons. To this end, instead of parametrising the gauge interactions via covariant derivatives, we shall instead use the notation of [55] and supplement our interactions (2.2) with 


$$
\begin{aligned}
\mathcal{L}_{\mathrm{SV}}= & -g^{a i j} A_{a}^{\mu} \phi_{i} \partial_{\mu} \phi_{j}-\frac{1}{4} g^{a b i j} A_{a}^{\mu} A_{\mu b} \phi_{i} \phi_{j} \\
& -\frac{1}{2} g^{a b i} A_{a}^{\mu} A_{\mu b} \phi_{i} \\
\mathcal{L}_{\mathrm{FV}}= & g_{I}^{a J} A_{a}^{\mu} \psi^{\dagger I} \bar{\sigma}_{\mu} \psi_{J}
\end{aligned}
$$

with repeated indices summed over, and metric signature $(-+++)$.

$$
\begin{aligned}
V_{F F V}^{(2)} & =\frac{1}{2}\left|g_{I}^{a J}\right|^{2} f_{F F V}\left(m_{I}^{2}, m_{J}^{2}, m_{a}^{2}\right), \\
V_{\overline{F F} V}^{(2)} & =\frac{1}{2} g_{I}^{a J} g_{I^{\prime}}^{a J^{\prime}} M^{I I^{\prime}} M_{J J^{\prime}}^{*} f_{\overline{F F} V}\left(m_{I}^{2}, m_{J}^{2}, m_{a}^{2}\right), \\
V_{\text {gauge }}^{(2)} & =\frac{1}{12}\left(g^{a b c}\right)^{2} f_{\text {gauge }}\left(m_{a}^{2}, m_{b}^{2}, m_{c}^{2}\right) .
\end{aligned}
$$

\begin{tabular}{|c|c|c|}
\hline & $\overline{D R}^{\prime}$ & $\Delta_{\overline{M S}}$ \\
\hline$f_{S S V}$ & $\begin{array}{c}\frac{1}{z}\left[-\Delta(x, y, z) I(x, y, z)+(x-y)^{2} I(0, x, y)\right. \\
+(y-x-z) J(x, z)+(x-y-z) J(y, z)+z J(x, y)] \\
+2(x+y-z / 3) J(z)\end{array}$ & 0 \\
\hline$f_{V S}$ & $3 J(x, y)$ & $2 x J(y)$ \\
\hline$f_{V V S}$ & $\begin{array}{c}\frac{1}{4 x y}[(-\Delta(x, y, z)-12 x y) I(x, y, z) \\
+(x-z)^{2} I(0, x, z)+(y-x)^{2} I(0, y, z)-z^{2} I(0,0, z) \\
+(z-x-y) J(x, y)+y J(x, z)+x J(y, z)] \\
+\frac{1}{2} J(x)+\frac{1}{2} J(y)\end{array}$ & $2 J(z)-x-y-z$ \\
\hline$f_{F F V}$ & $\begin{array}{c}\frac{1}{z}\left[\left(\Delta(x, y, z)-3 z^{2}+3 x z+3 y z\right)\right) I(x, y, z)-(x-y)^{2} I(0, x, y) \\
+(x-y-2 z) J(x, z)+(y-x-2 z) J(y, z)+2 z J(x, y)] \\
+2(-x-y+z / 3) J(z)\end{array}$ & $-2 x J(x)-2 y J(y)+(x+y)^{2}-z^{2}$ \\
\hline$f_{\overline{F F} V}$ & $6 I(x, y, z)$ & $2(x+y+z)-4 J(x)-4 J(y)$ \\
\hline$f_{\text {gauge }}$ & $\begin{array}{c}\frac{1}{4 x y z}\left\{\left(-x^{4}-8 x^{3} y-8 x^{3} z+32 x^{2} y z+18 y^{2} z^{2}\right) I(x, y, z)\right. \\
+(y-z)^{2}\left(y^{2}+10 y z+z^{2}\right) I(0, y, z)+x^{2}\left(2 y z-x^{2}\right) I(0,0, x) \\
+\left(x^{2}-9 y^{2}-9 z^{2}+9 x y+9 x z+14 y z\right) x J(y, z) \\
+(22 y+22 z-40 x / 3) x y z J(x)\}+(x \leftrightarrow y)+(x \leftrightarrow z)\end{array}$ & $\begin{array}{l}x^{2}+12 y z+2 x J(x) \\
+(x \leftrightarrow y)+(x \leftrightarrow z)\end{array}$ \\
\hline
\end{tabular}

The modified loop functions are

\section{C.1: Effective potential}

The full two-loop effective potential in the Landau gauge was given in Ref. [55]:

$$
\begin{aligned}
V_{S S S}^{(2)} & =\frac{1}{12}\left(\lambda^{i j k}\right)^{2} f_{S S S}\left(m_{i}^{2}, m_{j}^{2}, m_{k}^{2}\right), \\
V_{S S}^{(2)} & =\frac{1}{8} \lambda^{i i j j} f_{S S}\left(m_{i}^{2}, m_{j}^{2}\right), \\
V_{F F S}^{(2)} & =\frac{1}{2}\left|y^{I J k}\right|^{2} f_{F F S}\left(m_{I}^{2}, m_{J}^{2}, m_{k}^{2}\right), \\
V_{\overline{F F} S}^{(2)} & =\frac{1}{4} y^{I J k} y^{I^{\prime} J^{\prime} k} M_{I I^{\prime}}^{*} M_{J J^{\prime}}^{*} f_{\overline{F F} S}\left(m_{I}^{2}, m_{J}^{2}, m_{k}^{2}\right)+\text { c.c. }, \\
V_{S S V}^{(2)} & =\frac{1}{4}\left(g^{a i j}\right)^{2} f_{S S V}\left(m_{i}^{2}, m_{j}^{2}, m_{a}^{2}\right), \\
V_{V S}^{(2)} & =\frac{1}{4} g^{a a i i} F_{V S}\left(m_{a}^{2}, m_{i}^{2}\right), \\
V_{V V S}^{(2)} & =\frac{1}{4}\left(g^{a b i}\right)^{2} f_{V V S}\left(m_{a}^{2}, m_{b}^{2}, m_{i}^{2}\right),
\end{aligned}
$$

where $\Delta(x, y, z) \equiv x^{2}+y^{2}+z^{2}-2 x y-2 x z-2 y z$ and it is understood that $f_{\alpha}^{\overline{M S}}=f_{\alpha} \overline{D R}^{\prime}+\Delta_{\overline{M S}}$.

\section{C.2: Tadpoles}

Clearly our results for the derivatives involving no vectors given in the text are unchanged. However, for all others we will have to apply our procedure to the more complicated loop functions and also the derivatives of the scalar masses and couplings. In fact, of the new couplings only $g^{a b i}$ has a non-trivial derivative, so we require

$$
\begin{aligned}
m_{a b}^{2}(S) \delta^{\mu v} & =-\frac{\partial^{2} \mathcal{L}}{\partial A_{\mu}^{a} \partial A_{v}^{a}} \\
m_{a b}^{2}(S) & =m_{a}^{2} \delta^{a b}+g^{a b i} S_{i}+\frac{1}{2} g^{a b i j} S_{i} S_{j} \\
g^{a b i}(S) & =g^{a b i}+g^{a b i j} S_{j},
\end{aligned}
$$

and therefore 
$\frac{\partial}{\partial S_{r}} g^{a b i}=g^{a b i r}, \frac{\partial}{\partial S_{r}} m_{a b}^{2}(S)=g^{a b r}(S)$

In the following we define

$$
\begin{aligned}
f_{\alpha}^{(1,0,0)}(x, u ; y, z) & \equiv \frac{f_{\alpha}(x, y, z)-f_{\alpha}(u, y, z)}{x-u}, \\
f_{\alpha}^{(0,0,1)}(x, y ; z, u) & \equiv \frac{f_{\alpha}(x, y, z)-f_{\alpha}(x, y, u)}{z-u},
\end{aligned}
$$

i.e. we can give the loop functions used for the tadpoles in terms of those in the effective potential. In general these can be simplified, in particular to allow the smooth limit $u \rightarrow x$ to be taken, but we postpone that to future work where we shall also treat the second derivatives. Here we simply present the full set of tadpole diagrams, modifying (2.30):

$$
\begin{aligned}
\frac{\partial V^{(2)}}{\partial S_{p}^{0}}= & R_{r p}^{0}\left[T_{S}+T_{S S F F}+T_{F F F S}+T_{S S V}+T_{V S}\right. \\
& \left.+T_{V V S}+T_{F F V}+T_{\overline{F F} V}+T_{\text {gauge }}\right]
\end{aligned}
$$

where $T_{S}, T_{S S F F}, T_{F F F S}$ are as given in the body of the paper and

$$
\begin{aligned}
& T_{S S V}=\frac{1}{2} g^{a i j} g^{a k j} \lambda^{i k r} f_{S S V}^{(1,0,0)}\left(m_{i}^{2}, m_{k}^{2} ; m_{j}^{2}, m_{a}^{2}\right) \\
& +\frac{1}{4} g^{a i j} g^{b i j} g^{a b r} f_{S S V}^{(0,0,1)}\left(m_{i}^{2}, m_{j}^{2} ; m_{a}^{2}, m_{b}^{2}\right), \\
& T_{S S V}=\frac{1}{4} g^{a b i i} g^{a b r} f_{V S}^{(1,0)}\left(m_{a}^{2}, m_{b}^{2} ; m_{i}^{2}\right) \\
& +\frac{1}{4} g^{\text {aaik }} \lambda^{i k r} f_{V S}^{(0,1)}\left(m_{a}^{2} ; m_{i}^{2}, m_{k}^{2}\right) \\
& T_{V V S}=\frac{1}{2} g^{a b i} g^{c b i} g^{a c r} f_{V V S}^{(1,0,0)}\left(m_{a}^{2}, m_{c}^{2} ; m_{b}^{2}, m_{i}^{2}\right) \\
& +\frac{1}{4} g^{a b i} g^{a b j} \lambda^{i j r} f_{V V S}^{(0,0,1)}\left(m_{a}^{2}, m_{b}^{2} ; m_{i}^{2}, m_{j}^{2}\right), \\
& T_{F F V}=2 g_{I}^{a J} \bar{g}_{b J}^{K} \operatorname{Re}\left(M_{K I^{\prime}} y^{I^{\prime} I r}\right) f_{F F V}^{(1,0,0)}\left(m_{I}^{2}, m_{K}^{2} ; m_{J}^{2}, m_{a}^{2}\right) \\
& +\frac{1}{2} g_{I}^{a J} \bar{g}_{b J}^{I} g^{a b r} f_{F F V}^{(0,0,1)}\left(m_{I}^{2}, m_{J}^{2} ; m_{a}^{2}, m_{b}^{2}\right), \\
& T_{\overline{F F} V}=g_{I}^{a J} g_{I^{\prime}}^{a J^{\prime}} \operatorname{Re}\left(y^{I I^{\prime} r} M_{J J^{\prime}}^{*}\right)\left[f_{\overline{F F} V}\left(m_{I}^{2}, m_{J}^{2}, m_{a}^{2}\right)\right. \\
& \left.+M_{I}^{2} f_{\frac{F F}{F}}^{(1,0,0)}\left(m_{I}^{2}, m_{I^{\prime}}^{2} ; m_{J}^{2}, m_{a}^{2}\right)\right] \\
& +g_{I}^{a J} g_{I^{\prime}}^{a J^{\prime}} \operatorname{Re}\left(M^{I K^{\prime}} M^{K I^{\prime}} M_{J J^{\prime}}^{*} y_{K K^{\prime} r}\right) \\
& \times f_{\overline{F F} V}^{(1,0,0)}\left(m_{I}^{2}, m_{I^{\prime}}^{2} ; m_{J}^{2}, m_{a}^{2}\right) \\
& +\frac{1}{2} g_{I}^{a J} g_{I^{\prime}}^{b J^{\prime}} g^{a b r} M^{I I^{\prime}} M_{J J^{\prime}}^{*} \\
& \times f_{\overline{F F} V}^{(0,0,1)}\left(m_{I}^{2}, m_{J}^{2} ; m_{a}^{2}, m_{b}^{2}\right), \\
& T_{\text {gauge }}=\frac{1}{4} g^{a b c} g^{d b c} g^{a d r} f_{\text {gauge }}^{(1,0,0)}\left(m_{a}^{2}, m_{d}^{2} ; m_{b}^{2}, m_{c}^{2}\right) \text {. }
\end{aligned}
$$

\section{References}

1. CMS Collaboration, S. Chatrchyan et al., Observation of a new boson at a mass of $125 \mathrm{GeV}$ with the CMS experiment at the LHC. Phys. Lett. B 716, 30-61 (2012). arXiv:1207.7235

2. ATLAS Collaboration, G. Aad et al., Observation of a new particle in the search for the Standard Model Higgs boson with the ATLAS detector at the LHC. Phys. Lett. B 716, 1-29 (2012). arXiv: 1207.7214

3. CMS Collaboration, V. Khachatryan et al., Observation of the diphoton decay of the Higgs boson and measurement of its properties. Eur. Phys. J. C 74(10), 3076 (2014). arXiv:1407.0558

4. F. Staub, SARAH. arXiv:0806.0538

5. F. Staub, From Superpotential to model files for FeynArts and CalcHep/CompHep. Comput. Phys. Commun. 181, 1077-1086 (2010). arXiv:0909.2863

6. F. Staub, Automatic calculation of supersymmetric renormalization group equations and self energies. Comput. Phys. Commun. 182, 808-833 (2011). arXiv: 1002.0840

7. F. Staub, SARAH 3.2: Dirac gauginos, UFO output, and more. Comput. Phys. Commun. 184, 1792-1809 (2013). arXiv:1207.0906

8. F. Staub, SARAH 4: a tool for (not only SUSY) model builders. Comput. Phys. Commun. 185, 1773-1790 (2014). arXiv: 1309.7223

9. W. Porod, SPheno, a program for calculating supersymmetric spectra, SUSY particle decays and SUSY particle production at e+ e- colliders. Comput. Phys. Commun. 153, 275-315 (2003). arXiv:hep-ph/0301101

10. W. Porod, F. Staub, SPheno 3.1: extensions including flavour, CPphases and models beyond the MSSM. Comput. Phys. Commun. 183, 2458-2469 (2012). arXiv: 1104.1573

11. B. Allanach, SOFTSUSY: a program for calculating supersymmetric spectra. Comput. Phys. Commun. 143, 305-331 (2002). arXiv:hep-ph/0104145

12. P. Athron, J.-H. Park, D. Stöckinger, A. Voigt, FlexibleSUSY A spectrum generator for supersymmetric models. Comput. Phys. Commun. 190, 139-172 (2015). arXiv:1406.2319

13. A. Brignole, Radiative corrections to the supersymmetric charged Higgs boson mass. Phys. Lett. B 277, 313-323 (1992)

14. P.H. Chankowski, S. Pokorski, J. Rosiek, Charged and neutral supersymmetric Higgs boson masses: Complete one loop analysis. Phys. Lett. B 274, 191-198 (1992)

15. A. Dabelstein, The one loop renormalization of the MSSM Higgs sector and its application to the neutral scalar Higgs masses. Z. Phys. C 67, 495-512 (1995). arXiv:hep-ph/9409375

16. D.M. Pierce, J.A. Bagger, K.T. Matchev, R.-J. Zhang, Precision corrections in the minimal supersymmetric standard model. Nucl. Phys. B 491, 3-67 (1997). arXiv:hep-ph/9606211

17. A. Pilaftsis, CP odd tadpole renormalization of Higgs scalar - pseudoscalar mixing. Phys. Rev. D 58, 096010 (1998). arXiv:hep-ph/9803297

18. A. Pilaftsis, Higgs scalar - pseudoscalar mixing in the minimal supersymmetric standard model. Phys. Lett. B 435, 88-100 (1998). arXiv:hep-ph/9805373

19. M. Frank, T. Hahn, S. Heinemeyer, W. Hollik, H. Rzehak et al., The Higgs boson masses and mixings of the complex MSSM in the Feynman-diagrammatic approach. JHEP 0702, 047 (2007). arXiv:hep-ph/0611326

20. G. Degrassi, P. Slavich, On the radiative corrections to the neutral Higgs boson masses in the NMSSM. Nucl. Phys. B 825, 119-150 (2010). arXiv:0907.4682

21. F. Staub, W. Porod, B. Herrmann, The electroweak sector of the NMSSM at the one-loop level. JHEP 1010, 040 (2010). arXiv: 1007.4049 
22. K. Ender, T. Graf, M. Muhlleitner, H. Rzehak, Analysis of the NMSSM Higgs boson masses at one-loop level. Phys. Rev. D 85, 075024 (2012). arXiv: 1111.4952

23. T. Graf, R. Grober, M. Muhlleitner, H. Rzehak, K. Walz, Higgs boson masses in the complex NMSSM at one-loop level. JHEP 1210, 122 (2012). arXiv: 1206.6806

24. S.P. Martin, Strong and Yukawa two-loop contributions to Higgs scalar boson self-energies and pole masses in supersymmetry. Phys. Rev. D 71, 016012 (2005). arXiv:hep-ph/0405022

25. S. Borowka, T. Hahn, S. Heinemeyer, G. Heinrich, W. Hollik, Momentum-dependent two-loop QCD corrections to the neutral Higgs-boson masses in the MSSM. Eur. Phys. J. C 74(8), 2994 (2014). arXiv:1404.7074

26. G. Degrassi, S. Di Vita, P. Slavich, Two-loop QCD corrections to the MSSM Higgs masses beyond the effective-potential approximation. Eur. Phys. J. C 75(2), 61 (2015). arXiv:1410.3432

27. R. Hempfling, A.H. Hoang, Two loop radiative corrections to the upper limit of the lightest Higgs boson mass in the minimal supersymmetric model. Phys. Lett. B 331, 99-106 (1994). arXiv:hep-ph/9401219

28. M.S. Carena, M. Quiros, C. Wagner, Effective potential methods and the Higgs mass spectrum in the MSSM. Nucl. Phys. B 461, 407-436 (1996). arXiv:hep-ph/9508343

29. S. Heinemeyer, W. Hollik, G. Weiglein, QCD corrections to the masses of the neutral CP - even Higgs bosons in the MSSM. Phys. Rev. D 58, 091701 (1998). arXiv:hep-ph/9803277

30. R.-J. Zhang, Two loop effective potential calculation of the lightest CP even Higgs boson mass in the MSSM. Phys. Lett. B 447, 89-97 (1999). arXiv:hep-ph/9808299

31. S. Heinemeyer, W. Hollik, G. Weiglein, The masses of the neutral CP - even Higgs bosons in the MSSM: accurate analysis at the two loop level. Eur. Phys. J. C 9, 343-366 (1999). arXiv:hep-ph/9812472

32. S. Heinemeyer, W. Hollik, G. Weiglein, The mass of the lightest MSSM Higgs boson: a Compact analytical expression at the two loop level. Phys. Lett. B 455, 179-191 (1999). arXiv:hep-ph/9903404

33. J.R. Espinosa, R.-J. Zhang, MSSM lightest CP even Higgs boson mass to $\mathrm{O}($ alpha(s) alpha(t)): the effective potential approach. JHEP 0003, 026 (2000). arXiv:hep-ph/9912236

34. J.R. Espinosa, R.-J. Zhang, Complete two loop dominant corrections to the mass of the lightest $\mathrm{CP}$ even Higgs boson in the minimal supersymmetric standard model. Nucl. Phys. B 586, 3-38 (2000). arXiv:hep-ph/0003246

35. G. Degrassi, P. Slavich, F. Zwirner, On the neutral Higgs boson masses in the MSSM for arbitrary stop mixing. Nucl. Phys. B 611, 403-422 (2001). arXiv:hep-ph/0105096

36. A. Brignole, G. Degrassi, P. Slavich, F. Zwirner, On the $\mathrm{O}(\operatorname{alpha}(\mathrm{t}) * * 2)$ two loop corrections to the neutral Higgs boson masses in the MSSM. Nucl. Phys. B 631, 195-218 (2002). arXiv:hep-ph/0112177

37. A. Brignole, G. Degrassi, P. Slavich, F. Zwirner, On the two loop sbottom corrections to the neutral Higgs boson masses in the MSSM. Nucl. Phys. B 643, 79-92 (2002). arXiv:hep-ph/0206101

38. S.P. Martin, Two loop effective potential for the minimal supersymmetric standard model. Phys. Rev. D 66, 096001 (2002). arXiv:hep-ph/0206136

39. S.P. Martin, Complete two loop effective potential approximation to the lightest Higgs scalar boson mass in supersymmetry. Phys. Rev. D 67, 095012 (2003). arXiv:hep-ph/0211366

40. A. Dedes, P. Slavich, Two loop corrections to radiative electroweak symmetry breaking in the MSSM. Nucl. Phys. B 657, 333-354 (2003). arXiv:hep-ph/0212132

41. A. Dedes, G. Degrassi, P. Slavich, On the two loop Yukawa corrections to the MSSM Higgs boson masses at large tan beta. Nucl. Phys. B 672, 144-162 (2003). arXiv:hep-ph/0305127
42. S. Heinemeyer, W. Hollik, H. Rzehak, G. Weiglein, The Higgs sector of the complex MSSM at two-loop order: QCD contributions. Phys. Lett. B 652, 300-309 (2007). arXiv:0705.0746

43. W. Hollik, S. Paßehr, Higgs boson masses and mixings in the complex MSSM with two-loop top-Yukawa-coupling corrections. JHEP. 1410, 171 (2014). arXiv:1409.1687

44. M. Mühlleitner, D.T. Nhung, H. Rzehak, K. Walz, Two-loop contributions of the order $O\left(\alpha_{t} \alpha_{s}\right)$ to the masses of the Higgs bosons in the CP-violating NMSSM. JHEP. 1505, 128 (2015). arXiv: 1412.0918

45. S. Heinemeyer, W. Hollik, G. Weiglein, FeynHiggs: a program for the calculation of the masses of the neutral CP even Higgs bosons in the MSSM. Comput. Phys. Commun. 124, 76-89 (2000). arXiv:hep-ph/9812320

46. A. Djouadi, J.-L. Kneur, G. Moultaka, SuSpect: a fortran code for the supersymmetric and Higgs particle spectrum in the MSSM. Comput. Phys. Commun. 176, 426-455 (2007). arXiv:hep-ph/0211331

47. U. Ellwanger, C. Hugonie, NMSPEC: a fortran code for the sparticle and Higgs masses in the NMSSM with GUT scale boundary conditions. Comput. Phys. Commun. 177, 399-407 (2007). arXiv:hep-ph/0612134

48. B. Allanach, P. Athron, L.C. Tunstall, A. Voigt, A. Williams, Nextto-minimal SOFTSUSY. Comput. Phys. Commun. 185, 2322-2339 (2014). arXiv: 1311.7659

49. B. Allanach, A. Bednyakov, R. Ruiz de Austri, Higher order corrections and unification in the minimal supersymmetric standard model: SOFTSUSY3.5. Comput. Phys. Commun. 189, 192-206 (2015). arXiv:1407.6130

50. J. Baglio, R. Gröber, M. Mühlleitner, D. Nhung, H. Rzehak et al., NMSSMCALC: a program package for the calculation of loopcorrected Higgs boson masses and decay widths in the (complex) NMSSM. Comput. Phys. Commun. 185(12), 3372-3391 (2014). arXiv: 1312.4788

51. S.P. Martin, Three-loop standard model effective potential at leading order in strong and top Yukawa couplings. Phys. Rev. D 89, 013003 (2014). arXiv: 1310.7553

52. S.P. Martin, D.G. Robertson, Higgs boson mass in the standard model at two-loop order and beyond. Phys. Rev. D 90(7), 073010 (2014). arXiv:1407.4336

53. S.P. Martin, Three-loop corrections to the lightest Higgs scalar boson mass in supersymmetry. Phys. Rev. D 75, 055005 (2007). arXiv:hep-ph/0701051

54. P. Kant, R. Harlander, L. Mihaila, M. Steinhauser, Light MSSM Higgs boson mass to three-loop accuracy. JHEP 1008, 104 (2010). arXiv: 1005.5709

55. S.P. Martin, Two loop effective potential for a general renormalizable theory and softly broken supersymmetry. Phys. Rev. D 65, 116003 (2002). arXiv:hep-ph/0111209

56. S.P. Martin, Two loop scalar self energies in a general renormalizable theory at leading order in gauge couplings. Phys. Rev. D 70, 016005 (2004). arXiv:hep-ph/0312092

57. S.P. Martin, Evaluation of two loop selfenergy basis integrals using differential equations. Phys. Rev. D 68, 075002 (2003). arXiv:hep-ph/0307101

58. S.P. Martin, Two-loop scalar self-energies and pole masses in a general renormalizable theory with massless gauge bosons. Phys. Rev. D 71, 116004 (2005). arXiv:hep-ph/0502168

59. S.P. Martin, Taming the Goldstone contributions to the effective potential. Phys. Rev. D 90, 016013 (2014). arXiv:1406.2355

60. J. Elias-Miro, J. Espinosa, T. Konstandin, Taming infrared divergences in the effective potential. JHEP 1408, 034 (2014). arXiv: 1406.2652

61. M.D. Goodsell, K. Nickel, F. Staub, Two-loop Higgs mass calculations in supersymmetric models beyond the MSSM with SARAH and SPheno. Eur. Phys. J. C 75(1), 32 (2015). arXiv:1411.0675 
62. M.D. Goodsell, K. Nickel, F. Staub, Two-loop corrections to the Higgs masses in the NMSSM. Phys. Rev. D 91(3), 035021 (2015). arXiv: 1411.4665

63. S.P. Martin, D.G. Robertson, TSIL: a program for the calculation of two-loop self-energy integrals. Comput. Phys. Commun. 174, 133-151 (2006). arXiv:hep-ph/0501132

64. W. Porod, F. Staub, A. Vicente, A flavor kit for BSM models. Eur. Phys. J. C 74(8), 2992 (2014). arXiv: 1405.1434
65. M.D. Goodsell, P. Slavich, In prep

66. B. O'Leary, W. Porod, F. Staub, Mass spectrum of the minimal SUSY B-L model. JHEP 1205, 042 (2012). arXiv: 1112.4600

67. H.K. Dreiner, K. Nickel, F. Staub, On the two-loop corrections to the Higgs mass in trilinear R-parity violation. Phys. Lett. B 742, 261-265 (2015). arXiv:1411.3731 